\title{
THE ISOMORPHISM PROBLEM FOR TWO- GENERATOR ONE-RELATOR GROUPS WITH TORSION IS SOLVABLE
}

BY

\author{
STEPHEN J. PRIDE
}

\begin{abstract}
The theorem stated in the title is obtained by determining (in a sense to be made precise) all the generating pairs of an arbitrary twogenerator one-relator group with torsion. As a consequence of this determination it is also deduced that every two-generator one-relator group $G$ with torsion is Hopfian, and that the automorphism group of $G$ is finitely generated.
\end{abstract}

1. Introduction. The main aim of this paper is to establish

THEOREM 1. There is an algorithm to decide for any two presentations $\left\langle x_{1}, x_{2} ; P^{m}\right\rangle,\left\langle x_{1}, x_{2} ; Q^{n}\right\rangle$, where $\left.m, n\right\rangle 1$, whether or not the presentations define isomorphic groups.

This theorem is obtained as a consequence of the following lemma.

Let $G$ be a two-generator group. Recall [9] that two generating pairs $\left(g_{1}, g_{2}\right),\left(g_{1}^{\prime}, g_{2}^{\prime}\right)$ of $G$ are said to be Nielsen equivalent if there is an automorphism $x_{1} \mapsto Y_{1}\left(x_{1}, x_{2}\right), x_{2} \mapsto Y_{2}\left(x_{1}, x_{2}\right)$ of the free group $F_{2}$ on $x_{1}, x_{2}$ such that $g_{i}^{\prime}=Y_{i}\left(g_{1}, g_{2}\right)$ for $i=1,2$. Also, the pairs $\left(g_{1}, g_{2}\right),\left(g_{1}^{\prime}, g_{2}^{\prime}\right)$ are said to lie in the same $T$-system if there is an automorphism $\xi$ of $G$ such that $\left(g_{1}^{\prime}, g_{2}^{\prime}\right)$ is Nielsen equivalent to $\left(\xi\left(g_{1}\right), \xi\left(g_{2}\right)\right)$.

PRINCIPAl Lemma. Let $G=\left\langle a, t ; R^{n}\right\rangle$ where $R$ is not a true power, and where $n>1$. If $R$ is a primitive in the free group on $a, t$ then $G$ has one Nielsen equivalence class when $n=2$, or $\frac{1}{2} \varphi(n)$ Nielsen equivalence classes and one $T$ system when $n>2$. If $R$ is not a primitive then $G$ has one Nielsen equivalence class.

Here $\varphi$ denotes the Euler totient function.

To see how Theorem 1 follows from the Principal Lemma observe that by Lemma 1 of [9] and the Principal Lemma above, two presentations $\left\langle x_{1}, x_{2}\right.$; $\left.P^{m}\right\rangle,\left\langle x_{1}, x_{2} ; Q^{n}\right\rangle$, where $m, n>1$, are presentations of isomorphic groups if and only if there is an automorphism of $F_{2}$ mapping $P^{m}$ to $Q^{ \pm n}$. Since there

Received by the editors September 5, 1975.

AMS (MOS) subject classifications (1970). Primary 20E30, 20F05; Secondary $20 \mathrm{~F} 55$. 
is an algorithm to decide for any two elements $S$ and $T$ of $F_{2}$ whether or not $T$ is equal to the image of $S$ under an automorphism of $F_{2}$ (see Theorem N2 of [3]), Theorem 1 follows.

Apart from its use in proving Theorem 1, the Principal Lemma is also helpful in obtaining other information concerning two-generator one-relator groups with torsion.

Theorem 2. Let $G=\left\langle a, t ; R^{n}\right\rangle$ where $\left.n\right\rangle 1$. Then $G$ is Hopfian.

This is an immediate consequence of the Principal Lemma and Theorem 2 of [9].

THEOREM 3. Let $G=\left\langle a, t ; R^{n}\right\rangle$ where $\left.n\right\rangle 1$. Then the automorphism group, $\operatorname{Aut}(G)$, of $G$ is finitely generated( $(1)$.

This result is easily proved for the case when $R$ is a power of a primitive.

Suppose on the other hand, that $R$ is not a power of a primitive. Then $G$ has one Nielsen equivalence class by the Principal Lemma, so that every automorphism from an automorphism

$$
a \mapsto Y_{1}(a, t), \quad t \mapsto Y_{2}(a, t),
$$

where $\left(Y_{1}(a, t), Y_{2}(a, t)\right)$ is a generating pair of the free group $F$ on $a, t$ and where $R\left(Y_{1}(a, t), Y_{2}(a, t)\right)$ is equal in $F$ to either $R(a, t)$ or $R^{-1}(a, t)$. Now it is shown in [4] that the group of automorphisms of $F$ which map $R$ to $R^{ \pm 1}$ is finitely generated. Since the group of inner automorphisms of $G$ is also finitely generated, it follows that $\operatorname{Aut}(G)$ is finitely generated.

The present paper makes heavy use of results and techniques developed in [9] and [10]. The fact that one-relator groups are HNN groups will be made use of frequently throughout the paper, and the reader may like to consult the expository article [5] by McCool and Schupp to see how theorems concerning one-relator groups can be proved using the theory of HNN groups. The standard reference for notation and background material used throughout will be the book [3] by Magnus, Karrass and Solitar. Unexplained concepts and notation which cannot be found in [3] will be as in [9].

It is worthwhile to give here an outline of the proof of the Principal Lemma. The most difficult case to deal with is when $R$ is neither freely equal to 1 nor a primitive. To handle this case it is no loss of generality to assume that $R$ is cyclically reduced and involves $a, t$, and that the exponent sum of $R$ on $t$ is zero. Let $a_{i}(i=0, \pm 1, \pm 2, \ldots)$ denote the word $t^{-i} a t^{i}$, and let $P$ be the word obtained from $R$ by rewriting it in terms of the $a_{i}$. Let $m$ and $M$ be, respectively, the least and greatest integers $i$ for which $a_{i}$ occurs in $P$. Then, as

(1) J. $\mathrm{McCool}$ and I have recently established that $\mathrm{Aut}(G)$ is finitely presented. 
observed by Moldavanskii [6], $G$ can be presented as an HNN group as follows:

$$
G=\left\langle a_{m}, \ldots, a_{M}, t ; P^{n}, t^{-1} a_{i} t=a_{i+1}(i=m, \ldots, M-1)\right\rangle .
$$

Now the associated subgroups $K_{-1}=\operatorname{sgp}\left\{a_{m}, \ldots, a_{M-1}\right\}$ and $K_{1}$ $=\operatorname{sgp}\left\{a_{m+1}, \ldots, a_{M}\right\}$ are malnormal in the base $H=\left\langle a_{m}, \ldots, a_{M} ; P^{n}\right\rangle$, and so it follows from Theorem 6 of [9] that every generating pair of $G$ is Nielsen equivalent to a pair of the form $(t h, k)$ where $h$ and $k$ belong to $H$, and where $k$ is a nonempty cyclically reduced word in the generators of $K_{-1}$. Moreover $h k h^{-1} \notin K_{1}$.

Let $k^{(i)}(i=0,1, \ldots)$ denote the element $(t h)^{-i} k(t h)^{i}$. Then there is an integer $\lambda$ with $0<\lambda \leqslant M-m+1$ such that $k^{(i)} \in H$ if and only if $0 \leqslant i \leqslant \lambda$. Moreover $\lambda=M-m+1$ only if $k$ is a power of $a_{m}$. The main part of the proof is involved with showing that if $(t h, k)$ generates $G$ then $k^{(0)}, \ldots, k^{(\lambda)}$ generate $H$. For then, since $H$ cannot be generated by less than $M-m+1$ elements, it follows that $k=a_{m}^{l}$ for some integer $l$. It can then be established without too much difficulty that $\left(t h, a_{m}^{l}\right)$ generates $G$ if and only if $|l|=1$ and $t h=a_{m}^{\alpha} t a_{m}^{\beta}$ for suitable integers $\alpha, \beta$. Thus $(t h, k)$ is Nielsen equivalent to $\left(t, a_{0}\right)$, so that $G$ has one Nielsen equivalence class.

In order to show that $k^{(0)}, \ldots, k^{(\lambda)}$ generate $H$ whenever $t h, k$ generate $G$, it must be established that a word $W$ in $t h, k^{(0)}, \ldots, k^{(\lambda)}$ which defines an element of $H$ is equal to a word in $k^{(0)}, \ldots, k^{(\lambda)}$ alone. This is easily proved using Britton's lemma and induction on the $t$-length of $W$ once the following formulae have been established:

$$
\begin{gathered}
\operatorname{sgp}\left\{k^{(0)}, \ldots, k^{(\lambda)}\right\} \cap K_{-1}=\operatorname{sgp}\left\{k^{(0)}, \ldots, k^{(\lambda-1)}\right\}, \\
h \operatorname{sgp}\left\{k^{(0)}, \ldots, k^{(\lambda)}\right\} h^{-1} \cap K_{1}=h \operatorname{sgp}\left\{k^{(1)}, \ldots, k^{(\lambda)}\right\} h^{-1} .
\end{gathered}
$$

These formulae follow from Theorem 3 of [10] when $\lambda>1$. For if $\lambda>1$ then $h \in K_{-1}$ and $\left(h k^{(0)} h^{-1}, \ldots, h k^{(\lambda)} h^{-1}\right)$ is $\left(a_{m}, a_{M}\right)$-admissible. However, when $\lambda=1$, Theorem 3 of [10] is not necessarily applicable. All one knows in general in this case is that $k^{(0)} \in K_{-1}, k^{(1)} \notin K_{-1}, h k^{(0)} h^{-1} \notin K_{1}, h k^{(1)} h^{-1}$ $\in K_{1}$. Consequently it is necessary to establish that if $u \in K_{\varepsilon}(|\varepsilon|=1)$ and $v \notin K_{\varepsilon}$ then $\operatorname{sgp}\{u, v\} \cap K_{\varepsilon}=\operatorname{sgp}\{u\}$. In actual fact, it becomes necessary to prove a more general result than this so that the usual inductive techniques for dealing with one-relator groups can be used.

Let $B=\left\langle x_{j}(j \in J) ; S, T, \ldots\right\rangle$ and for $j \in J$ define $L_{j}$ to be the subgroup of $B$ generated by those generators of $B$ other than $x_{j}$. Then $B$ (or more precisely this presentation of $B$ ) will be said to have property-I provided the following holds: for each $j$ in $J$, if $u \in L_{j}$ and $v \notin L_{j}$ then $\operatorname{sgp}\{u, v\} \cap L_{j}$ $=\operatorname{sgp}\{u\}$. It will be shown below that 
The remainder of the paper is divided into three sections. In $\$ 2$ various concepts and definitions are introduced and several useful lemmas, mainly concerning HNN groups, are obtained. In $\$ 3$ a proof of (*) is given. $\$ 4$ investigates the generating pairs of an arbitrary two-generator one-relator group with torsion, culminating in a proof of the Principal Lemma. Each of $\S \S 2,3,4$ is subdivided and has an introduction explaining its contents more fully.

The techniques developed in this paper will be used in a future article to describe the two-generator subgroups of an arbitrary one-relator group with torsion.

2. Preliminaries. In $\$ 2.1$ the basic notation and definitions needed for the rest of the paper are introduced. It is shown how to present a one-relator group, whose defining relator has zero sum-exponent on some generator, as an HNN group, the base $H$ of which is another one-relator group. Several lemmas concerning such an HNN group are then obtained. In $\$ 2.2$ the definition is given of standard H-elements (of which the $k^{(i)}$ in the previous section are examples). These elements are then analysed in some detail.

2.1. Definitions, notation, and some lemmas. Throughout the paper $\varepsilon$ (or some variation such as $\varepsilon^{\prime}, \varepsilon_{1}, \varepsilon_{2}$ ) will denote an integer of modulus 1 . The set of integers will be denoted by $\mathbf{Z}$. If $\nu$ is a real number [ $\nu]$ will denote the greatest integer less than or equal to $\nu$.

If $G$ is a group and $u, v \in G$ then the element $u^{-1} v u$ of $G$ will be denoted by $u^{\nu}$, and will be called the conjugate of $u$ by $v$. If $A$ is a subset of $G$ then the subgroup of $G$ generated by $A$ will be denoted by $\operatorname{sgp} A$. By convention, if $A$ is empty then $\operatorname{sgp} A$ is the trivial subgroup 1 .

Let $G=\left\langle a, c, d, \ldots, t ; R^{n}\right\rangle$ where $\left.n\right\rangle 1, R$ is a cyclically reduced word which involves $a, \sigma_{t}(R)=0$. Let $\alpha$ be the maximum of the set

\section{$\{\tau: \tau$ is the exponent sum on $t$ of an initial segment}

$$
\text { of } R \text { which precedes an } a \text {-symbol\}. }
$$

Then clearly $G=\left\langle a, c, d, \ldots, t ; t^{-\alpha} R^{n} t^{\alpha}\right\rangle$. For $i \in \mathbf{Z}$ let $a_{i}, c_{i}, d_{i}, \ldots$ denote the words $t^{-i} a t^{i}, t^{-i} c t^{i}, t^{-i} d t^{i}, \ldots$ respectively. Then $t^{-\alpha} R t^{\alpha}$ can be rewritten as a cyclically reduced word $P$ in the $a_{i}, c_{i}, d_{i}, \ldots$ as follows. Replace a symbol $x^{\varepsilon}$, where $x$ is one of $a, c, d, \ldots$, which appears in $t^{-\alpha} R t^{\alpha}$ by $x_{-i}^{\varepsilon}$, where $i$ is the exponent sum on $t$ of the initial segment of $t^{-\alpha} R t^{\alpha}$ preceding $x^{\varepsilon}$. Then clearly $a_{0}$ appears in $P$. The largest integer $i$ for which $a_{i}$ appears in $P$ will be denoted by $M$. Notice that $P$ involves at least one generator having a nonzero subscript if and only if $R$ involves $t$. Notice 
also that if $R$ involves $t$ then the length of $P$ is less than the length of $R$.

Now it is not difficult to show using Tietze transformations that

$$
\begin{aligned}
G=\left\langle a_{0}, \ldots, a_{M}, c_{i}(i \in \mathbf{Z}), d_{i}(i \in \mathbf{Z}), \ldots, t ; P^{n},\right. \\
t^{-1} a_{0} t=a_{1}, \ldots, t^{-1} a_{M-1} t=a_{M}, \\
\left.t^{-1} c_{i} t=c_{i+1}(i \in \mathbf{Z}), t^{-1} d_{i} t=d_{i+1}(i \in \mathbf{Z}), \ldots\right\rangle .
\end{aligned}
$$

Let

$$
\begin{aligned}
H & =\left\langle a_{0}, \ldots, a_{M}, c_{i}(i \in \mathbf{Z}), d_{i}(i \in \mathbf{Z}), \ldots ; P^{n}\right\rangle, \\
K_{-1} & =\operatorname{sgp}\left\{a_{0}, \ldots, a_{M-1}, c_{i}(i \in \mathbf{Z}), d_{i}(i \in \mathbf{Z}), \ldots\right\}
\end{aligned}
$$

and

$$
K_{1}=\operatorname{sgp}\left\{a_{1}, \ldots, a_{M}, c_{i}(i \in \mathbf{Z}), d_{i}(i \in \mathbf{Z}), \ldots\right\} .
$$

Then $K_{-1}$ and $K_{1}$ are free on the given generators (by the Freiheitssatz), and so $G$ is presented above as an HNN group with base $H$, stable letter $t$, and associated subgroups $K_{-1}$ and $K_{1}$. This HNN presentation of $G$ will be called the $H N N$ presentation of $G$ with stable letter $t$ and fixed generator $a$.

It should be noted that $P, M, H, K_{-1}, K_{1}$ are all dependent on $R, a, t$, but to avoid cumbersome notation (such as $P(R, a, t), M(R, a, t)$, etc.) this dependence will not be made explicit. This should cause no confusion.

It was first observed by Moldavanskii [6] that if $A$ is a one-relator group whose defining relator $Q$ is cyclically reduced and has exponent sum zero on some generator occuring in it, then $A$ is an HNN extension of another onerelator group whose defining relator is shorter than $Q$. This observation was taken up by $\mathrm{McCool}$ and Schupp [5] and others to give rather elegant induction proofs of the basic results on one-relator groups. Such induction techniques will be employed here. However, it is not always necessary to use induction to obtain results about one-relator groups. In some cases it suffices to know that the group is a nontrivial HNN extension of another one-relator group $B$ and that the associated subgroups lie "suitably" in $B$. This was the approach adopted in [11] for example, and such an approach will also be used here.

Basic facts concerning HNN groups which will be needed in the sequel can be found in $[9, \S \S 1.2,2.1]$. Additional results will be obtained below.

It is worthwhile to make some comments concerning $t$-reducing in the HNN group $G$ above. Suppose $w$ is a word in the generators of $K_{-e^{\prime}}$. Then the $t$ reduced form $w^{\prime}$ of $t^{-\varepsilon} w t^{\ell}$ is obtained from $w$ by replacing each occurrence of a generator $x_{i}$, where $x$ is one of $a, c, d, \ldots$, by $x_{i+e}$ (such a procedure is called 
"shifting subscripts" in [5]). Now clearly $w t^{\varepsilon}=t^{\varepsilon} w^{\prime}$. It follows that if $W$ is a word $w_{0} t^{\varepsilon_{1}} w_{1} \cdots t^{\varepsilon_{r}} w_{r}$ where the $w_{j}$ are words in $c_{i}(i \in \mathbf{Z}), d_{i}(i \in \mathbf{Z}), \ldots$, then the $t$-symbols can be "pulled through" either all to the left or all to the right, so that there are words $u$ and $v$ in $c_{i}(i \in \mathbf{Z}), d_{i}(i \in \mathbf{Z}), \ldots$ such that $W=t^{s} u=v t^{s}$, where $s=\sum_{j=1}^{r} \varepsilon_{j}$.

Several results relevant to the HNN group $G$ above will now be obtained.

The first lemma is required since the associated subgroups $K_{-1}$ and $K_{1}$ are free. The lemma is easily proved.

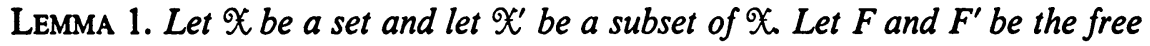
groups on $\mathcal{X}$ and $\mathfrak{X}^{\prime}$ respectively. Suppose $A \subseteq F^{\prime}$ and $v \in F \backslash F^{\prime}$. Then:

(i) $F^{\prime} \cap \operatorname{sgp} A \cup\{v\}=\operatorname{sgp} A$;

(ii) if $\operatorname{sgp} A$ is free on $A$ then $\operatorname{sgp} A \cup\{v\}$ is free on $A \cup\{v\}$.

EXAMPLE. Let $k$ be a nonempty freely reduced word in $c_{i}(i \in \mathbf{Z})$, $d_{i}(i \in \mathbf{Z}), \ldots$, and for $i \in \mathbf{Z}$ let $k^{(i)}$ denote the $t$-reduced form of $t^{-i} k t^{i}$. Let $q$ and $s$ be respectively the lowest and highest integers $j$ for which $x_{j}$ (where $x$ is one of $c, d, \ldots)$ occurs in $k$, and for $l, m \geqslant 0$, let $X_{l, m}=\left\{c_{i}, d_{i}, \ldots: q\right.$ $-l \leqslant i \leqslant s+m\}$ and let $F_{l, m}$ be the free group on $\mathscr{X}_{l, m}$. Then

$$
F_{0,0} \subset F_{0,1} \subset F_{1,1} \subset F_{1,2} \subset F_{2,2} \subset \cdots .
$$

Moreover, if $\mu>0$ then

$$
\left\{k^{(0)}, k^{(1)}, k^{(-1)}, \ldots, k^{(\mu-1)}, k^{(-\mu+1)}\right\} \subseteq F_{\mu-1, \mu-1}
$$

whereas $k^{(\mu)} \in F_{\mu-1, \mu} \backslash F_{\mu-1, \mu-1}$, and

$$
\left\{k^{(0)}, k^{(1)}, k^{(-1)}, \ldots, k^{(\mu-1)}, k^{(-\mu+1)}, k^{(\mu)}\right\} \subseteq F_{\mu-1, \mu}
$$

whereas $k^{(-\mu)} \in F_{\mu, \mu} \backslash F_{\mu-1, \mu}$. Thus by repeated use of Lemma 1(ii) it is deduced that the $k^{(i)}(i \in Z)$ freely generate a subgroup of $\operatorname{sgp}\left\{c_{i}(i \in Z)\right.$, $\left.d_{i}(i \in \mathbf{Z}), \ldots\right\}$.

As well as being free, the groups $K_{-1}$ and $K_{1}$ have several other useful properties, which for convenience are listed here.

(2.1) $K_{-1}$ and $K_{1}$ are malnormal in $H$.

See Lemma 2.1 of [8]. Recall that a subgroup $B$ of a group $A$ is said to be malnormal in $A$ if, for all $g \in A, g^{-1} B g \cap B \neq 1$ implies $g \in B$.

(2.2) $A$ freely reduced (respectively, cyclically reduced) word in the generators of $K_{-1}$ which involves $a_{0}$ is not equal (resp. conjugate in $H$ ) to an element of $K_{1}$, and a freely reduced (resp. cyclically reduced) word in the generators of $K_{1}$ which involves $a_{M}$ is not equal (resp. conjugate in $H$ ) to an element of $K_{-1}$. 
The result for freely reduced words follows from Newman's Spelling Theorem (Theorem 3 of [7]). The result for cyclically reduced words is obtained as follows. Suppose for definiteness that $k$ is a cyclically reduced word in the generators of $K_{-1}$ and that $k$ involves $a_{0}$ (notice that this implies $M>0$ ). It must be established that an equation $h^{-1} k h=u$, where $u$ is a cyclically reduced word in the generators of $K_{1}$ and where $h \in H$, is impossible. This follows from Lemma 2.1 of [8] if $u$ involves $a_{M}$. On the other hand, if $u$ does not involve $a_{M}$ then $u \in K_{-1}$, so that $h \in K_{-1}$ by (2.1). Thus the equation takes place in the free group $K_{-1}$, which once again is impossible.

Let $u, w_{i}(i \in I), v$ be elements of $H$ and let $\left(u, w_{i}(i \in I), v\right)$ denote an $(|I|+2)$-tuple with $u$ in the first position, $v$ in the last position, and the $w_{i}$ listed in some order. The tuple will be called weakly $\left(a_{0}, a_{M}\right)$-admissible if $u \in K_{-1} \backslash K_{1}, v \in K_{1} \backslash K_{-1}$ and $w_{i} \in K_{-1} \cap K_{1}$ for $i \in I$. If in addition the $w_{i}$ freely generate a subgroup of $K_{-1} \cap K_{1}$ then the tuple will be called $\left(a_{0}, a_{M}\right)$-admissible. The concept of an $\left(a_{0}, a_{M}\right)$-admissible tuple of words in the generators of $H$ was introduced in [10]. It is easily seen using (2.2) that if the tuple $\left(u, w_{i}(i \in I), v\right)$ is $\left(a_{0}, a_{M}\right)$-admissible in the sense just defined, and if $u, w_{i}(i \in I), v$ are expressed as words in the generators of $K_{-1}$ or $K_{1}$ (whichever is appropriate) then the resulting tuple is $\left(a_{0}, a_{M}\right)$-admissible in the sense of [10]. Conversely an $\left(a_{0}, a_{M}\right)$-admissible tuple in the sense of [10] is $\left(a_{0}, a_{M}\right)$-admissible in the sense just defined, by (2.2).

(2.3) If $\left(u, w_{i}(i \in I), v\right)$ is weakly $\left(a_{0}, a_{M}\right)$-admissible then

$$
\operatorname{sgp}\left\{u, w_{i}(i \in I), v\right\} \cap K_{-1}=\operatorname{sgp}\left\{u, w_{i}(i \in I)\right\}
$$

and

$$
\operatorname{sgp}\left\{u, w_{i}(i \in I), v\right\} \cap K_{1}=\operatorname{sgp}\left\{w_{i}(i \in I), v\right\}
$$

This follows immediately from Theorem 3 of [10] (taking account of the previous discussion) in the case when $\left(u, w_{i}(i \in I), v\right)$ is $\left(a_{0}, a_{M}\right)$-admissible. But obviously the fact that the $w_{i}$ freely generate a subgroup of $K_{-1} \cap K_{1}$ is immaterial.

LEMMA 2. Let $Z$ be a $t$-reduced word and let $h$ be a $t$-free word. Suppose there is an integer $m_{0}$ such that $h^{m_{0}} \neq 1$ and $Z^{-1} h^{m_{0}} Z$ is not $t$-reduced. Then $Z^{-1} h^{m} Z$ is not $t$-reduced for any integer $m$.

This is simply a special case of Lemma 9 of [9], taking account of (2.1).

LEMMA 3. Let $k$ be a freely reduced word in the generators of $K_{\varepsilon}$ and assume $t^{-r} k t^{r}$ defines an element of $H$.

(i) Suppose $k$ involves an $a_{i}$-symbol and let $q$ and $s$ be respectively the least and greatest integers $i$ for which $a_{i}$ occurs in $k$. Then $-q \leqslant r \leqslant M-s$. 
(ii) The t-reduced form of $t^{-r} k t^{r}$ is obtained from $k$ by replacing each generator $x_{i}$ (where $x$ is one of $a, c, d, \ldots$ ) appearing in $k$ by $x_{i+r}$.

Proof. (i) Suppose $r<-q$. Let $k^{*}$ be the word obtained from $k$ by replacing each generator $x_{i}$ (where $x$ is one of $a, c, d, \ldots$ ) appearing in $k$ by $x_{i-q}$. Then $k^{*}$ is the $t$-reduced form of $t^{q} k t^{-q}$ and $t^{-r} k t^{r}=t^{-(r+q)} k^{*} t^{r+q}$. Now $k^{*}$ is a word in the generators of $K_{-1}$ which involves $a_{0}$, and so it follows from (2.2) that $t^{-(r+q)} k^{*} t^{r+q}$ is $t$-reduced. Consequently $t^{-r} k t^{r}$ does not define an element of $H$, contrary to assumption.

In a similar way, if $r>M-s$ then the $t$-reduced form of $t^{-r} k t^{r}$ involves $t$ and therefore does not define an element of $H$.

(ii) The result is immediate if $k$ does not involve an $a_{i}$-symbol. Otherwise the result follows from (i).

LEMMA 4. Let $r$ be an integer.

If $|r|>M$ then $t^{r} H t^{-r} \cap H=\operatorname{sgp}\left\{c_{i}(i \in \mathbf{Z}), d_{i}(i \in \mathbf{Z}), \ldots\right\}$.

If $0 \leqslant r \leqslant M$ then $t^{r} H t^{-r} \cap H=\operatorname{sgp}\left\{a_{0}, \ldots, a_{M-r}, c_{i}(i \in \mathbf{Z}), d_{i}(i\right.$ $\in \mathbf{Z}), \ldots\}$.

If $-M \leqslant r \leqslant 0$ then $t^{r} H t^{-r} \cap H=\operatorname{sgp}\left\{a_{-r}, \ldots, a_{M}, c_{i}(i \in \mathbf{Z}), d_{i}(i\right.$ $\in \mathbf{Z}), \ldots\}$.

PRoof. The result is trivial if $r=0$.

Suppose $r>0$, and let $h \in t^{r} H t^{-r} \cap H$. Then it follows from Britton's lemma that there is a freely reduced word $k$ in the generators of $K_{1}$ such that $t^{r} k t^{-r}=h$. If $k$ does not involve an $a_{i}$-symbol then clearly $h \in \operatorname{sgp}\left\{c_{i}(i \in \mathbf{Z})\right.$, $\left.d_{i}(i \in Z), \ldots\right\}$. Suppose $k$ involves an $a_{i}$-symbol and let $q$ be the least integer $i$ for which $a_{i}$ occurs in $k$. Then $r \leqslant q$ by Lemma 3(ii), and the $t$-reduced form of $t^{r} k t^{-r}$ is a word in $a_{0}, \ldots, a_{M-r}, c_{i}(i \in \mathbf{Z}), d_{i}(i \in \mathbf{Z}), \ldots$ This shows that $t^{r} H t^{-r} \cap H$ is contained in $\operatorname{sgp}\left\{a_{0}, \ldots, a_{M-r}, c_{i}(i \in \mathbf{Z}), d_{i}(i \in \mathbf{Z}), \ldots\right\}$ if $r \leqslant q$, and is contained in $\operatorname{sgp}\left\{c_{i}(i \in \mathbf{Z}), d_{i}(i \in \mathbf{Z}), \ldots\right\}$ otherwise. The reverse inclusions are obvious.

The case when $r<0$ is handled similarly.

LEMMA 5. Let $Z \in G$ and let $k$ be a nonempty cyclically reduced word in the generators of $K_{e^{\prime}}$. If $Z^{-1} k Z \in H$ then $Z=t^{r} h$ for some integer $r$ and some element $h$ of $H$.

Proof. Let $V$ be an element of minimal $t$-length from the set

$\left\{U: U\right.$ is a $t$-reduced word equal to $t^{l} Z$ for some integer $\left.l\right\}$.

Then $Z=t^{r} V$ for some integer $r$, and $t^{r} V$ is $t$-reduced. It will be shown that $V$ is $t$-free. Suppose not, and let $V \equiv v t^{\delta} V^{\prime}$ where $v$ is $t$-free and $\delta= \pm 1$. It suffices to establish that $v \in K_{-\delta}$. For then $t^{-\delta} v t^{\delta}$ is equal to a $t$-free word $u$ 
and $t^{-(r+\delta)} Z=u V^{\prime}$, which contradicts the minimality of $V$. Now $t^{-r} k t^{r}$ defines an element of $H$, and so it follows from Lemma 3(ii) that the $t$-reduced form $k^{*}$ of $t^{-r} k t^{r}$ is a nonempty cyclically reduced word in the generators of one of $K_{-1}, K_{1}$. Moreover since $Z^{-1} k Z \in H, v^{-1} k^{*} v \in K_{-\delta}$. Thus $k^{*}$ $\in K_{-\delta}$ by (2.2), and so $v \in K_{-\delta}$ by (2.1).

LeMma 6. Suppose $R$ involves $t$ and $M=0$. Let $k$ be a cyclically reduced word in $a_{0}, c_{0}, d_{0}, \ldots$ which involves $a_{0}$, and let $Z$ be a t-reduced word which involves t. Then $Z^{-1} k^{m} Z(m \neq 0)$ is t-reduced.

Proof. It suffices, by Lemma 2 , to show that $Z^{-1} k Z$ is $t$-reduced. Suppose $Z$ has initial segment $z t^{\varepsilon}$, where $z$ is $t$-free, and assume by way of contradiction that

$$
z^{-1} k z \in \operatorname{sgp}\left\{c_{i}(i \in \mathbf{Z}), d_{i}(i \in \mathbf{Z}), \ldots\right\} \quad\left(=K_{-1}=K_{1}\right) .
$$

Then $t^{-\varepsilon} z^{-1} k z t^{\ell}$ is equal to a word in $c_{i}(i \in \mathbf{Z}), d_{i}(i \in \mathbf{Z}), \ldots$. Passing back to the one-relator presentation of $G$ it is thus concluded that there is a cyclically reduced word in $a, c, d, \ldots$ involving $a$ which is conjugate to a word which does not involve $a$. But an argument similar to that used to establish (2.2) shows that this is impossible.

2.2. Standard $H$-elements. Throughout this subsection $G, H, K_{-1}, K_{1}$ etc., will be as in $\$ 2.1$.

Let $p$ be a positive integer and let $u, v \in H$. For $i \in \mathbf{Z}$ let $v^{(i)}$ denote the element $\left(t^{p} u\right)^{-i} v\left(t^{p} u\right)^{i}$ of $G$. Those elements $v^{(i)}$ which belong to $H$ will be called the standard $H$-elements associated with $\left(t^{p} u, v\right)$ (or simply the standard $H$-elements if $\left(t^{p} u, v\right)$ is understood). Where necessary (for instance when using Britton's lemma) it will be assumed that the standard $H$-elements are written in terms of the generators of $H$.

It is clear from Britton's lemma that if $v^{(i)} \in H$ for some $i>0(i$ $<0)$ then $v^{(j)} \in H$ whenever $0 \leqslant j \leqslant i(i \leqslant j \leqslant 0)$.

The standard $H$-elements can be thought of as the "obvious" elements of $H$ which can be obtained from $t^{p} u, v$. The reason for considering these elements stems from their importance in calculating the intersection of $\operatorname{sgp}\left\{t^{p} u, v\right\}$ with $H$. The determination of such an intersection is a key step in the proof of the Principal Lemma.

There are two main situations where standard $H$-elements arise in the sequel.

(A) Let $v$ be a nonempty cyclically reduced word in the generators of $K_{-1}$ and let $u$ be a $t$-free word. Suppose that not both of $u, v$ belong to $\operatorname{sgp}\left\{c_{i}(i \in \mathbf{Z}), d_{i}(i \in \mathbf{Z}), \ldots\right\}$ and consider the pair $\left(t^{p} u, v\right), p>0$.

Now up to conjugation by a power of $t$ it can be assumed that $u v u^{-1} \notin K_{1}$. Indeed, suppose $u v u^{-1} \in K_{1}$. Then it follows from (2.2) that $v$ does not 
involve $a_{0}$, so that $v \in K_{1}$. Thus $u \in K_{1}$ by (2.1). Assume that $u$ is written as a freely reduced word in the generators of $K_{1}$ and let $q$ be the least integer $i$ for which $a_{i}$ occurs in one of $u, v$. Let $\bar{u}, \bar{v}$ be the $t$-reduced forms of $t^{q} u t^{-q}, t^{q} v t^{-q}$ respectively. Then $t^{q} t^{p} u t^{-q}=t^{p} \bar{u}$. Moreover $\bar{v}$ is cyclically reduced. Now $\bar{u} \bar{v} \bar{u}^{-1} \notin K_{1}$. For since $\bar{v}$ is cyclically reduced and $\bar{u}, \bar{v}$ are freely reduced words in the generators of $K_{-1}$, it follows that the freely reduced form of $\bar{u} \bar{v} \bar{u}^{-1}$ involves $a_{0}$, and therefore does not define an element of $K_{1}$ by (2.2). Assume from now on that $u v u^{-1} \notin K_{1}$.

Suppose $v^{(\mu)} \in H$ for some positive integer $\mu$. Then it follows from Lemma 5 that $t^{-\mu p}\left(t^{p} u\right)^{\mu} \in H$. Consequently $u, u^{t p}, \ldots, u^{t^{(\mu-1) p}} \in H$ and

$$
v^{(\mu)}=u^{-1} u^{-t^{p}} \cdots u^{-t^{(\mu-1) p}} v^{t^{\mu p}} u^{t^{(\mu-1) p}} \cdots u^{t^{p}} u .
$$

This implies that there is an integer $\lambda$ such that $v^{(i)} \in H$ if and only if $0 \leqslant i \leqslant \lambda$. For if $v^{t^{\mu p}}$ and $u^{t^{(\mu-1) p}}$ belonged to $H$ for infinitely many values of $\mu$ then $v$ and $u$ would both belong to $\operatorname{sgp}\left\{c_{i}(i \in \mathbf{Z}), d_{i}(i \in \mathbf{Z}), \ldots\right\}$ by Lemma 4, contrary to assumption.

Now since $v^{t^{\lambda p}} \in H$, it follows from Lemma 3(i) that $\lambda p \leqslant M$, and so for $j=0,1, \ldots, \lambda$ one can consider the subgroup $F^{(j)}$ of $H$ generated by $a_{0}, \ldots, a_{M-(\lambda-j) p}, c_{i}(i \in \mathbf{Z}), d_{i}(i \in \mathbf{Z}), \ldots$. It will be shown that if $1 \leqslant \mu$ $\leqslant \lambda$ then $v^{(\mu)} \in F^{(\mu)} \backslash F^{(\mu-1)}$.

Now $u \in t^{(\lambda-1) p} H t^{-(\lambda-1) p} \cap H$, and

$$
t^{(\lambda-1) p} H t^{-(\lambda-1) p} \cap H=\operatorname{sgp}\left\{a_{0}, \ldots, a_{M-(\lambda-1) p}, c_{i}(i \in \mathbf{Z}), d_{i}(i \in \mathbf{Z}), \ldots\right\}
$$

by Lemma 4 . Thus:

$$
u^{t^{l p}} \in F^{(l+1)}, \quad l=0,1, \ldots, \lambda-1 .
$$

In a similar way:

$$
v^{l^{l p}} \in F^{(l)}, \quad l=0,1, \ldots, \lambda .
$$

It then follows from (2.4)-(2.6) that $v^{(\mu)} \in F^{(\mu)}$.

It is clear from the definition of $\lambda$ that $v^{(\lambda)} \notin F^{(\lambda-1)}$. Suppose by way of contradiction that for some integer $\mu$, with $1 \leqslant \mu<\lambda, v^{(\mu)} \in F^{(\mu-1)}$. Now

$$
\begin{aligned}
& v^{(\lambda)}=\left(t^{p} u\right)^{-(\lambda-\mu)} v^{(\mu)}\left(t^{p} u\right)^{\lambda-\mu}
\end{aligned}
$$

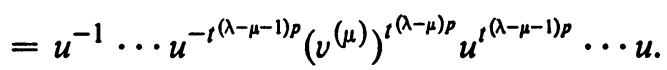

Observe that $u^{u^{(\lambda-\mu-1) p}} \cdots u \in F^{(\lambda-\mu)}$ by (2.5). Moreover, since $v^{(\mu)} \in F^{(\mu-1)}$ it

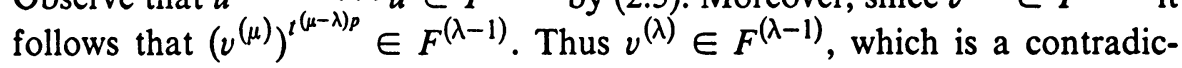
tion. 
(B) Let $z$ be a freely reduced word in the generators of one of $K_{-1}, K_{1}$ and suppose $z$ involves an $a_{i}$-symbol. Let $\left\{k_{j}: j \in J\right\}$ be a set of elements of the subgroup $F^{(-1)}$ of $H$ generated by $c_{i}(i \in \mathbf{Z}), d_{i}(i \in \mathbf{Z}), \ldots$, and suppose the $k_{j}$ freely generate a subgroup of $F^{(-1)}$. Suppose further that there is a permutation $\psi$ of $J$ such that, for each $j$ in $J, t^{-1} k_{j} t=k_{\psi(j)}$ (in other words, $\left\{k_{j}: j \in J\right\}$ is closed under conjugation by $\left.t\right)$. Consider the collection $t, z$, $k_{j}(j \in J)$. As in (A), up to conjugation by a power of $t$, it can be assumed that $z \in K_{-1} \backslash K_{1}$. Let $z^{(0)}, \ldots, z^{(\lambda)}$ be the standard $H$-elements associated with $(t, z)$. Then clearly $\left(z^{(0)}, z^{(1)}, \ldots, z^{(\lambda-1)}, k_{j}(j \in J), z^{(\lambda)}\right)$ is weakly $\left(a_{0}\right.$, $a_{M}$ )-admissible. Moreover, $z^{(0)}, z^{(1)}, \ldots, z^{(\lambda-1)}, k_{j}(j \in J)$ freely generate a subgroup of $K_{-1}$, so that in particular $\left(z^{(0)}, z^{(1)}, \ldots, z^{(\lambda-1)}, k_{j}(j \in J), z^{(\lambda)}\right)$ is $\left(a_{0}, a_{M}\right)$-admissible. To see that $z^{(0)}, z^{(1)}, \ldots, z^{(\lambda-1)}, k_{j}(j \in J)$ freely generate a subgroup of $K_{-1}$ let

$$
F^{(\mu)}=\operatorname{sgp}\left\{a_{0}, \ldots, a_{M-(\lambda-\mu)}, c_{i}(i \in \mathbf{Z}), d_{i}(i \in \mathbf{Z}), \ldots\right\}
$$

for $\mu=0,1, \ldots, \lambda$. Then $\left\{z^{(0)}, \ldots, z^{(\mu-1)}, k_{j}(j \in J)\right\} \subseteq F^{(\mu-1)}$, whereas $z^{(\mu)}$ $\in F^{(\mu)} \backslash F^{(\mu-1)}$ (see (A)). Then the result follows by repeated use of Lemma 1(ii).

Now

$$
\begin{gathered}
\operatorname{sgp}\left\{z^{(0)}, z^{(1)}, \ldots, z(\lambda-1), k_{j}(j \in J), z^{(\lambda)}\right\} \cap K_{-1} \\
=\operatorname{sgp}\left\{z^{(0)}, z^{(1)}, \ldots, z^{(\lambda-1)}, k_{j}(j \in J)\right\}
\end{gathered}
$$

and

$$
\begin{array}{r}
\operatorname{sgp}\left\{z^{(0)}, z^{(1)}, \ldots, z^{(\lambda-1)}, k_{j}(j \in J), z^{(\lambda)}\right\} \cap K_{1} \\
=\operatorname{sgp}\left\{z^{(1)}, \ldots, z^{(\lambda-1)}, k_{j}(j \in J), z^{(\lambda)}\right\},
\end{array}
$$

by (2.3). Using these formulae it will be deduced that

$$
\operatorname{sgp}\left\{t, z, k_{j}(j \in J)\right\} \cap H=\operatorname{sgp}\left\{z^{(0)}, \ldots, z^{(\lambda)}, k_{j}(j \in J)\right\} .
$$

To prove (2.9) it suffices to show that a word $W$ in $t, z^{(0)}, \ldots, z^{(\lambda)}, k_{j}(j \in J)$ which defines an element of $H$ is equal to a word in $z^{(0)}, \ldots, z^{(\lambda)}, k_{j}(j \in J)$ alone. The proof is by induction on the number of occurrences of $t$ in $W$.

If there are none the result holds.

Suppose then that $W$ involves $t$ and that $W$ defines an element of $H$. Then it follows from Britton's lemma that $W$ has a subword $t^{-\varepsilon} Q t^{\ell}$, where $Q$ is a word in $z^{(0)}, \ldots, z^{(\lambda)}, k_{j}(j \in J)$ and $Q$ defines an element of $K_{-\varepsilon}$. Now by (2.7) and (2.8), $Q$ is equal to another word $Q^{\prime}$ in $z^{(0)}, \ldots, z^{(\lambda)}, k_{j}(j \in J)$, 
where $Q^{\prime}$ does not involve either $z^{(\lambda)}, z^{(0)}$ according as $\varepsilon$ is $1,-1$. Thus $t^{-\varepsilon} Q t^{e}$ is equal to a word $S$ in $z^{(0)}, \ldots, z^{(\lambda)}, k_{j}(j \in J)$, where $S$ does not involve either $z^{(0)}, z^{(\lambda)}$ according as $\varepsilon$ is $1,-1$. Replacing $t^{-\varepsilon} Q t^{\varepsilon}$ by $S$ then gives a word $W^{\prime}$ in $t, z^{(0)}, \ldots, z^{(\lambda)}, k_{j}(j \in J)$ equal to $W$ in $G$ but having less occurrences of $t$. The inductive hypothesis can now be applied to give the desired conclusion. This completes the verification of (2.9).

It is possible to generalize (2.9). Indeed, for $\mu=-1,0, \ldots, \lambda$ :

$$
\operatorname{sgp}\left\{t, z, k_{j}(j \in J)\right\} \cap F^{(\mu)}=\operatorname{sgp}\left\{z^{(0)}, \ldots, z^{(\mu)}, k_{j}(j \in J)\right\} .
$$

To prove this, note that for $i=0, \ldots, \lambda-1,\left\{z^{(0)}, \ldots, z^{(i-1)}, k_{j}(j \in J)\right\}$ $\subseteq F^{(i-1)}$ whereas $z^{(i)} \in F^{(i)} \backslash F^{(i-1)}$ (see (A)), so it follows from Lemma $1(\mathrm{i})$ that

$$
\begin{gathered}
\operatorname{sgp}\left\{z^{(0)}, \ldots, z^{(i-1)}, z^{(i)}, k_{j}(j \in J)\right\} \cap F^{(i-1)} \\
=\operatorname{sgp}\left\{z^{(0)}, \ldots, z^{(i-1)}, k_{j}(j \in J)\right\} .
\end{gathered}
$$

This formula is also valid for $i=\lambda$, being in that case merely a restatement of (2.7). Combining (2.11) and (2.9) establishes that (2.10) holds.

Finally, a presentation of $\operatorname{sgp}\left\{t, z, k_{j}(j \in J)\right\}$ associated with the generators $t, z^{(0)}, \ldots, z^{(\lambda)}, k_{j}(j \in J)$ is obtained as follows. By Theorem 1 of [10] every relation between $z^{(0)}, \ldots, z^{(\lambda)}, k_{j}(j \in J)$ is a consequence of a single relation

$$
Q^{n}=1
$$

say, where $Q$ is either empty or is a cyclically reduced word involving $z^{(0)}$ and $z^{(\lambda)}$. Then an argument similar to that used to derive (2.9) from (2.7) and (2.8) can be employed to show that every relation between $t, z^{(0)}, \ldots, z^{(\lambda)}$, $k_{j}(j \in J)$ is a consequence of (2.12) and the additional relations:

$$
t^{-1} z^{(0)} t=z^{(1)}, \ldots, t^{-1} z^{(\lambda-1)} t=z^{(\lambda)}, \quad t^{-1} k_{j} t=k_{\psi(j)} \quad(j \in J) .
$$

3. Intersections. The main aim of this section is to establish the following theorem.

THEOREM 4. Let $B=\left\langle x, y, b, \ldots ; R^{n}\right\rangle$ where $\left.n\right\rangle$ 1. Then $B$ has property-I.

This theorem will be proved by induction on the length of $R$, making use of the fact that if the cyclically reduced form of $R$ involves at least two generators then $B$ can be embedded into an HNN group whose base is a one-relator group, the relator of which has length less than $L(R)$. The following two results will therefore be useful.

Let 


$$
L=\left\langle a_{0}, \ldots, a_{N}, c_{i}(i \in \mathbf{Z}), d_{i}(i \in \mathbf{Z}), \ldots ; Q^{n}\right\rangle,
$$

where $N \geqslant 0, n>1, Q$ is a cyclically reduced word which involves $a_{0}$ and $a_{N}$. Let $G$ be the HNN group given by

$$
\begin{array}{r}
G=\left\langle a_{0}, \ldots, a_{N}, c_{i}(i \in \mathbf{Z}), d_{i}(i \in \mathbf{Z}), \ldots, t ; Q^{n}\right. \\
t^{-1} a_{i} t=a_{i+1}(i=0, \ldots, N-1), t^{-1} c_{i} t=c_{i+1}(i \in \mathbf{Z}), \\
\left.t^{-1} d_{i} t=d_{i+1}(i \in \mathbf{Z}), \ldots\right\rangle
\end{array}
$$

Suppose that $L$ has property-I.

(†) If $u$ is a cyclically reduced word in $a_{0}, c_{0}, d_{0}, \ldots$ which involves $a_{0}$, and if $v \notin \operatorname{sgp}\left\{a_{0}, c_{0}, d_{0}, \ldots\right\}$ then $\operatorname{sgp}\{u, v\} \cap \operatorname{sgp}\left\{a_{0}, c_{0}, d_{0}, \ldots\right\}=\operatorname{sgp}\{u\}$.

(See Proposition 2.)

( $\ddagger$ If $u$ is a nonempty freely reduced word in $t, c_{0}, d_{0}, \ldots$ and if $v$ $\notin \operatorname{sgp}\left\{t, c_{0}, d_{0}, \ldots\right\}$ then $\operatorname{sgp}\{u, v\} \cap \operatorname{sgp}\left\{t, c_{0}, d_{0}, \ldots\right\}=\operatorname{sgp}\{u\}$.

(See Proposition 3.)

Making use of $(\dagger)$ and $(\ddagger)$ it will now be shown how to prove Theorem 4 by induction on $L(R)$. It can be assumed without loss of generality that $R$ is cyclically reduced.

If $L(R)=0$ then $B$ is freely generated by $x, y, b, \ldots$ and the result is easily established.

Now suppose that $L(R)>0$. Let $u$ be a freely reduced word in $y, b, \ldots$, and suppose $v \notin \operatorname{sgp}\{y, b, \ldots\}$. It will be shown that $\operatorname{sgp}\{u, v\} \cap \operatorname{sgp}\{y$, $b, \ldots\}=\operatorname{sgp}\{u\}$. It suffices to consider the situation where $u$ is a cyclically reduced word in $y, b, \ldots$.

Case $1: x$ does not occur in $R$.

Then $B$ is the free product of the free group on $x$ and the one-relator group generated by the remaining generators. The result thus follows easily using the theory of free products.

Case 2: No generator occurring in $u$ also occurs in $R$.

Let $F$ denote the free group on those generators which occur in $u$, and let $B^{\prime}$ be the one-relator group generated by the remaining generators of $B$. Then $B$ is the free product of $F$ and $B^{\prime}$. Suppose $v=f_{0} g_{1} f_{1} \cdots g_{l} f_{l}$ where $l>0$, the $g_{i}$ are nontrivial elements of $B^{\prime}$, the $f_{i}$ are elements of $F$, nontrivial except possibly for $f_{0}$ and $f_{l}$. By assumption, at least one of the $g_{i}$ is equal to an element $g$ not belonging to $\operatorname{sgp}\{y, b, \ldots\}$.

Now the result is easily established if $f_{l} u^{p} f_{0} \neq 1$ for all integers $p$. Suppose on the other hand that $f_{l} u^{p} f_{0}=1$ for some integer $p$. Then it will be shown 
that $f_{0}^{-1} \operatorname{sgp}\left\{u, v u^{p}\right\} f_{0} \cap \operatorname{sgp}\{y, b, \ldots\}=f_{0}^{-1} \operatorname{sgp}\{u\} f_{0}$, from which it follows immediately that $\operatorname{sgp}\{u, v\} \cap \operatorname{sgp}\{y, b, \ldots\}=\operatorname{sgp}\{u\}$.

Now there is an integer $j$ with $0 \leqslant j \leqslant l-1$ such that if $1 \leqslant i \leqslant j$ then the $i$ th term of $g_{1} f_{1} \cdots g_{l}$ is the inverse of the $(2 l-i)$ th term, but the $(j+1)$ st term is not the inverse of the $(2 l-(j+1))$ st term if $j<l-1$. Let $T$ be the product of the first $j$ terms of $g_{1} f_{1} \cdots g_{l}$ (taken in order) and let $S$ be the product of the next $2(l-j)-1$ terms, so that $g_{1} f_{1} \cdots g_{l}$ and $T S T^{-1}$ are the same normal form. Now it is clear that the normal form of a product

$$
T S^{q_{0}} T^{-1}\left(f_{0}^{-1} u f_{0}\right)^{p_{1}} T S^{q_{1}} T^{-1} \cdots\left(f_{0}^{-1} u f_{0}\right)^{p_{r}} T S^{q_{r}} T^{-1}
$$

-where $r \geqslant 0$, the $\left|q_{i}\right|$ are nonzero and less than the order of $S$, the $\left|p_{i}\right|$ are nonzero and less than the order of $u$-has $g$ as one of its terms (and therefore does not define an element of $\operatorname{sgp}\{y, b, \ldots\})$ except possibly if $g$ is not one of the terms of $T$ and $S=g$. To see that the product does not define an element of $\operatorname{sgp}\{y, b, \ldots\}$ in this case, observe that since $\operatorname{sgp}\{y, b, \ldots\} \cap B^{\prime}$ is malnormal in $B^{\prime}$ (see [8, Lemma 2.1]), if $g^{q} \neq 1$ for some integer $q$ then $g^{q} \notin \operatorname{sgp}\{y, b, \ldots\}$. Thus the above product is in normal form and each of its terms $S^{q_{i}}$ lies outside $\operatorname{sgp}\{y, b, \ldots\}$.

Case 3: $x$ occurs in $R$ with zero-sum exponent; one of the generators occurs in both $u$ and $R$.

Suppose for definiteness that $y$ occurs in $u$ and $R$. Consider the HNN presentation of $B$ with stable letter $x$ and fixed generator $y$. By the inductive hypothesis the base of $B$ has property-I, so it follows from $(\dagger)$ that $\operatorname{sgp}\{u, v\}$ $\cap \operatorname{sgp}\{y, b, \ldots\}=\operatorname{sgp}\{u\}$.

Case 4: $x$ occurs in $R$; one of the generators which occurs in $u$ occurs in $R$ with zero-sum exponent.

Suppose $y$ occurs in $u$ and $R$, and $\sigma_{y}(R)=0$. Consider the HNN presentation of $B$ with stable letter $y$ and fixed generator $x$. Then the base has propertyI by the inductive hypothesis, so that the result follows from ( $\$$ ).

Case 5: $x$ occurs in $R ; \sigma_{x}(R) \neq 0$; one of the generators which occurs in $u$ occurs in $R$ with non zero-sum exponent.

Suppose for definiteness that $y$ occurs in $u$ and $R$, and $\sigma_{y}(R) \neq 0$. Let $\alpha=\sigma_{x}(R), \beta=\sigma_{y}(R)$. Let $B_{1}=\left\langle t, a, b, \ldots ; R_{1}^{n}\right\rangle$, where $R_{1}$ is the word obtained from $R$ by replacing each occurrence of $x$ by $a t^{-\beta}$ and each occurrence of $y$ by $t^{\alpha}$, and then cyclically reducing. Then $B$ is embedded into $B_{1}$ by the homomorphism $\Psi$ defined by

$$
x \mapsto a t^{-\beta}, \quad y \mapsto t^{\alpha}, \quad b \mapsto b, \ldots
$$

Moreover:

$$
\operatorname{sgp}\{t, b, \ldots\} \cap \Psi(B)=\operatorname{sgp}\left\{t^{\alpha}, b, \ldots\right\} .
$$


Now $R_{1}$ certainly involves $a$, and moreover $\sigma_{t}\left(R_{1}\right)=0$. Thus one can consider the HNN presentation of $B_{1}$ with stable letter $t$ and fixed generator $a$. The base of $B_{1}$ is another one-relator group, the relator of which has length less than $L(R)$. Consequently the base has property I by the inductive hypothesis. Now $\Psi(v) \notin \operatorname{sgp}\{t, b, \ldots\}$ by (3.3), and so it follows from ( $\ddagger$ ) that $\operatorname{sgp}\{\Psi(u), \Psi(v)\} \cap \operatorname{sgp}\{t, b, \ldots\}=\operatorname{sgp}\{\Psi(u)\}$. Thus

$$
\operatorname{sgp}\{u, v\} \cap \operatorname{sgp}\{y, b, \ldots\}=\operatorname{sgp}\{u\} .
$$

The above cases cover all possibilities and so the induction step is proved. In the following subsections statements $(\dagger)$ and $(\$)$ will be verified, and other results of a similar nature will also be obtained. For the remainder of this section $L$ and $G$ will be as in (3.1), (3.2). The associated subgroups $\operatorname{sgp}\left\{a_{0}, \ldots, a_{N-1}\right.$, $\left.c_{i}(i \in \mathbf{Z}), d_{i}(i \in \mathbf{Z}), \ldots\right\}$ and $\operatorname{sgp}\left\{a_{1}, \ldots, a_{N}, c_{i}(i \in \mathbf{Z}), d_{i}(i \in \mathbf{Z}), \ldots\right\}$ of $G$ will be denoted by $A_{-1}$ and $A_{1}$ respectively. It will be assumed throughout that $L$ has property-I.

3.1 Intersections of certain subgroups (1).

Proposition 1. Let $p$ be a positive integer, let $k$ be a nonempty cyclically reduced word in the generators of $A_{-1}$, and let $h$ be a $t$-free word. Assume that $h k h^{-1} \notin A_{1}$, and let $k^{(0)}, \ldots, k^{(\lambda)}$ be the standard L-elements. Then:

(i) $\operatorname{sgp}\left\{k^{(0)}, \ldots, k^{(\lambda)}\right\} \cap t^{p} L t^{-p}=\operatorname{sgp}\left\{k^{(0)}, \ldots, k^{(\lambda-1)}\right\}$;

(ii) $h \operatorname{sgp}\left\{k^{(0)}, \ldots, k^{(\lambda)}\right\} h^{-1} \cap A_{1}=h \operatorname{sgp}\left\{k^{(1)}, \ldots, k^{(\lambda)}\right\} h^{-1}$;

(iii) $\operatorname{sgp}\left\{t^{p} h, k\right\} \cap L=\operatorname{sgp}\left\{k^{(0)}, \ldots, k^{(\lambda)}\right\}$.

Consider (i). Suppose first that $k^{(0)}, \ldots, k^{(\lambda)}$ all belong to $A_{-1}$ and let $F=t^{p} L t^{-p} \cap L$. Then it follows from Lemma 4 that $F$ is freely generated by a subset of the generators of $A_{-1}$. Now $k^{(0)}, \ldots, k^{(\lambda-1)} \in F$ whereas $k^{(\lambda)}$ $\in A_{-1} \backslash F$, so it follows from Lemma $1(\mathrm{i})$ that $\operatorname{sgp}\left\{k^{(0)}, \ldots, k^{(\lambda)}\right\} \cap F$ $=\operatorname{sgp}\left\{k^{(0)}, \ldots, k^{(\lambda-1)}\right\}$, as required.

Now suppose that $k^{(\lambda)} \notin A_{-1}$ (note then that $\lambda>0$ ). If $\lambda>1$ then $h \in$ $A_{-1}($ see $\$ 2.2(\mathrm{~A}))$ so that $\left(h k^{(0)} h^{-1}, \ldots, h k^{(\lambda)} h^{-1}\right)$ is weakly $\left(a_{0}, a_{N}\right)$-admissible. Consequently

$$
h \operatorname{sgp}\left\{k^{(0)}, \ldots, k^{(\lambda)}\right\} h^{-1} \cap A_{-1}=h \operatorname{sgp}\left\{k^{(0)}, \ldots, k^{(\lambda-1)}\right\} h^{-1},
$$

by (2.3). Conjugating this equation by $h$ then gives

$$
\operatorname{sgp}\left\{k^{(0)}, \ldots, k^{(\lambda)}\right\} \cap A_{-1}=\operatorname{sgp}\left\{k^{(0)}, \ldots, k^{(\lambda-1)}\right\} .
$$

Observe that (3.4) is also valid if $\lambda=1$, since $L$ has property-I. Now $t^{p} L t^{-p} \cap L \subseteq A_{-1}$ and $\operatorname{sgp}\left\{k^{(0)}, \ldots, k^{(\lambda-1)}\right\} \subseteq t^{p} L t^{-p} \cap L$ so that (i) follows by intersecting both sides of (3.4) with $t^{p} L t^{-p} \cap L$.

Now consider (ii). If $\lambda=0$ the result follows from (2.1). If $\lambda=1$ the result 
follows from the fact that $L$ has property-I, for $h k^{(0)} h^{-1} \notin A_{1}$ whereas $h k^{(1)} h^{-1} \in A_{1}$. Suppose $\lambda>1$. Then $h \in A_{-1}$ so that $h k^{(0)} h^{-1} \in A_{-1} \backslash A_{1}$ and $h k^{(i)} h^{-1} \in A_{-1} \cap A_{1}$ for $i=1, \ldots, \lambda-1$. Thus if $h k^{(\lambda)} h^{-1} \in A_{1} \backslash A_{-1}$ the result follows from (2.3). Suppose on the other hand that $h k^{(\lambda)} h^{-1}$ $\in A_{-1} \cap A_{1}$. Then no element of $h \operatorname{sgp}\left\{k^{(0)}, \ldots, k^{(\lambda)}\right\} h^{-1}$ can be equal to a freely reduced word in the generators of $A_{1}$ which involves $a_{N} ;$ by (2.2). Consequently

$$
\begin{aligned}
h \operatorname{sgp}\left\{k^{(0)}, \ldots, k^{(\lambda)}\right\} h^{-1} & \cap A_{1}=h \operatorname{sgp}\left\{k^{(0)}, \ldots, k^{(\lambda)}\right\} h^{-1} \\
& \cap \operatorname{sgp}\left\{a_{1}, \ldots, a_{N-1}, c_{i}(i \in \mathbf{Z}), d_{i}(i \in \mathbf{Z}), \ldots\right\} .
\end{aligned}
$$

But it follows from Lemma $1(i)$ that

$$
\begin{gathered}
h \operatorname{sgp}\left\{k^{(0)}, \ldots, k^{(\lambda)}\right\} h^{-1} \cap \operatorname{sgp}\left\{a_{1}, \ldots, a_{N-1}, c_{i}(i \in \mathbf{Z}), d_{i}(i \in \mathbf{Z}), \ldots\right\} \\
=h \operatorname{sgp}\left\{k^{(1)}, \ldots, k^{(\lambda)}\right\} h^{-1} .
\end{gathered}
$$

To prove (iii) it must be established that if $W$ is a word in $t^{p} h, k^{(0)}, \ldots$, $k^{(\lambda)}$ which defines an element of $L$ then $W$ is equal to a word in $k^{(0)}, \ldots, k^{(\lambda)}$ alone. The proof is by induction on the number of occurrences of $t^{p} h$ in $W$.

If there are none the result holds.

Suppose that $W$ involves $t^{p} h$ and that $W$ defines an element of $L$. Now if $W$ has subword $t^{p} h T h^{-1} t^{-p}$ where $T$ is a word in $k^{(0)}, \ldots, k^{(\lambda)}$ and where $h T h^{-1} \in A_{1}$ then it follows from (ii) that $T$ is equal to a word in $k^{(1)}, \ldots$, $k^{(\lambda)}$. Consequently the subword $t^{p} h T h^{-1} t^{-p}$ can be replaced by a word in $k^{(0)}, \ldots, k^{(\lambda-1)}$ to give a word $W^{\prime}$ equal to $W$ in $G$ and where $W^{\prime}$ has less occurrences of $t^{p} h$. The inductive hypothesis can then be applied. Suppose on the other hand that $W$ does not have any subword $t^{p} h T h^{-1} t^{-p}$ as above. Then it follows from Britton's lemma that $W$ must have at least one subword of the form $h^{-1} t^{-p} S t{ }^{p} h$ where $S$ is a word in $k^{(0)}, \ldots, k^{(\lambda)}$ and $S \in A_{-1}$. Moreover, for at least one such subword, $S$ must belong to $t^{p} L t^{-p}$. For if this were not the case then the $t$-reduced form of every subword $h^{-1} t^{-p} S t^{p} h$ would involve $t$, so that the $t$-reduced form of $W$ would involve $t$, contrary to the fact that $W$ defines an element of $L$. Suppose then that $h^{-1} t^{-p} S t{ }^{p} h$ is a subword of $W$, where $S$ is a word in $k^{(0)}, \ldots, k^{(\lambda)}$ which defines an element of $t^{p} L t^{-p}$. Then it follows from (i) that $S$ is equal to a word in $k^{(0)}, \ldots, k^{(\lambda-1)}$ so that $h^{-1} t^{-p} S t^{p} h$ can be replaced by a word in $k^{(1)}, \ldots, k^{(\lambda)}$ and the inductive hypothesis can be applied to the resulting word.

This completes the proof of the proposition.

The following corollary to the proof of (iii) will be needed later.

COROLlary. Let $W$ be a word in $t^{p} h, k^{(0)}, \ldots, k^{(\lambda)}$. Then either $W$ is equal to a word $W^{\prime}$ in $t^{p} h, k^{(0)}, \ldots, k^{(\lambda)}$ where $W^{\prime}$ has less occurrences of $t^{p} h$ than 
$W$, or else in t-reducing $W$ at least one $t$-symbol from each subword $\left(t^{p} h\right)^{ \pm 1}$ remains.

\subsection{Intersections of certain subgroups (2).}

Proposition 2. Let $u$ be a cyclically reduced word in $a_{0}, c_{0}, d_{0}, \ldots$ which involves $a_{0}$, and let $v$ be an element of $G$ which does not belong to $\operatorname{sgp}\left\{a_{0}, c_{0}\right.$, $\left.d_{0}, \ldots\right\}$. Then $\operatorname{sgp}\{u, v\} \cap \operatorname{sgp}\left\{a_{0}, c_{0}, d_{0}, \ldots\right\}=\operatorname{sgp}\{u\}$.

If $Q$ does not involve any generator having a nonzero subscript then $G=\left\langle a_{0}, c_{0}, d_{0}, \ldots ; Q^{n}\right\rangle *\langle t\rangle$, and the result is easily established using the theory of free products. From now on therefore, it will be assumed that $Q$ involves at least one generator having a nonzero subscript.

Let $Z$ be an element of minimal $t$-length from the set

\section{$\left\{V: V\right.$ is the cyclically $t$-reduced form of $v u^{l}$ for some integer $\left.l\right\}$.}

Then there is an integer $m$ and a $t$-reduced word $T$ such that $v u^{m}=T Z T^{-1}$, and $T Z T^{-1}$ is $t$-reduced. It suffices to show that $\operatorname{sgp}\left\{T Z T^{-1}, u\right\} \cap \operatorname{sgp}\left\{a_{0}, c_{0}\right.$, $\left.d_{0}, \ldots\right\}=\operatorname{sgp}\{u\}$.

If for every integer $s, T u^{s} T^{-1}$ has $t$-reduced form of $t$-length greater than zero then $\operatorname{sgp}\left\{T Z T^{-1}, u\right\} \cap L=\operatorname{sgp}\{u\}$, so the result is clear.

Suppose on the other hand that $T^{-1} u^{s} T$ defines an element of $L$ for some nonzero integer $s$. Then it follows from Lemmas 5, 6 and 3(i) that $T=t^{r} g$ where $0 \leqslant r \leqslant N$ and $g$ is $t$-free. Replacing $Z$ by $g g^{-1}$ if necessary it can be supposed that $g$ is empty. It thus suffices to show that $\operatorname{sgp}\left\{Z, u_{r}\right\} \cap \operatorname{sgp}\left\{a_{r}\right.$, $\left.c_{r}, d_{r}, \ldots\right\}=\operatorname{sgp}\left\{u_{r}\right\}$. Here $u_{r}$ is the $t$-reduced form of $t^{-r} u t^{r}$ (that is, $u_{r}$ is the word obtained from $u$ by replacing $a_{0}$ by $a_{r}, c_{0}$ by $c_{r}, d_{0}$ by $\left.d_{r}, \ldots\right)$.

If $Z$ is $t$-free then the result follows from the fact that $L$ has property-I, for $Z \notin \operatorname{sgp}\left\{a_{r}, c_{r}, d_{r}, \ldots\right\}$.

Suppose $Z$ involves $t$. Then it follows from the definition of $Z$ that $Z u_{r}^{l} Z$ is $t$-reduced for all integers $l$. It is necessary to investigate the $t$-reductions of words $Z^{-1} u_{r}^{l} Z$ and $Z u_{r}^{j} Z^{-1}$ where $l, j$ are nonzero integers. By Lemma 2 it is enough to investigate the $t$-reductions of $Z^{-1} u_{r} Z$ and $Z u_{r} Z^{-1}$. Suppose that neither of $Z^{-1} u_{r} Z, Z u_{r} Z^{-1}$ is $t$-reduced. Let $Z$ have initial segment $z t^{\ell}$ and terminal segment $t^{\delta} w$. Here $\delta= \pm 1$ and $z, w$ are $t$-free. Then it follows from Lemmas 5 and 6 that $N>0$ and there are $t$-free words $z_{1}, w_{1}$ such that $z t^{\ell}=t^{e} z_{1}$ and $t^{\delta} w=w_{1} t^{\delta}$. Consequently $\varepsilon=\delta$ since $Z$ is cyclically $t$-reduced. However $\varepsilon \neq \delta$. This is clear if $r>0$ and $t^{r} Z t^{-r}$ is $t$-reduced. On the other hand, if $r=0$ then since by assumption $t^{-\varepsilon} u_{r} t^{\varepsilon}$ and $t^{\delta} u_{r} t^{-\delta}$ both define elements of $L$, equality of $\varepsilon$ and $\delta$ would imply $u_{r} \in A_{1}$, contrary to (2.2).

It has now been established that one of $Z^{-1} u_{r} Z, Z u_{r} Z^{-1}$ is $t$-reduced. By inverting $Z$ if necessary it can be supposed that $Z u_{r} Z^{-1}$ is $t$-reduced. Then 
$Z u_{r}^{j} Z^{-1}$ is $t$-reduced for all nonzero integers $j$, by Lemma 2 . It is thus easy to see that if the $t$-reduced form of $Z^{-1} u_{r}^{l} Z$ involves $t$ for every nonzero integer $l$ then a freely reduced word in $Z, u_{r}$ which involves $Z$ has $t$-reduced form of $t$-length greater than zero. Consequently $\operatorname{sgp}\left\{Z, u_{r}\right\} \cap L=\operatorname{sgp}\left\{u_{r}\right\}$, so that $\operatorname{sgp}\left\{Z, u_{r}\right\} \cap \operatorname{sgp}\left\{a_{r}, c_{r}, d_{r}, \ldots\right\}=\operatorname{sgp}\left\{u_{r}\right\}$ as required.

Now suppose that $Z^{-1} u_{r}^{l} Z$ defines an element of $L$ for some nonzero integer $l$. Then it follows from Lemmas 5,6 and 3(i) that $N>0$ and $Z=t^{p} h$, where $h$ is $t$-free and $0<p \leqslant N-r$ ( $p$ cannot be negative since $t^{r} Z t^{-r}$ is $t$ reduced). Let $u_{r}^{(0)}, \ldots, u_{r}^{(\lambda)}$ be the standard $L$-elements associated with $\left(t^{p} h, u_{r}\right)$. For $j=0, \ldots, \lambda$ let $F^{(j)}$ denote the subgroup of $L$ generated by $a_{0}, \ldots, a_{N-(\lambda-j) p}, c_{i}(i \in \mathbf{Z}), d_{i}(i \in \mathbf{Z}), \ldots$ Then

$$
\operatorname{sgp}\left\{t^{p} h, u_{r}\right\} \cap F^{(\lambda)}=\operatorname{sgp}\left\{u_{r}^{(0)}, \ldots, u_{r}^{(\lambda)}\right\}
$$

by Proposition 1(iii). Also:

$$
\begin{array}{r}
\operatorname{sgp}\left\{u_{r}^{(0)}, \ldots, u_{r}^{(j-1)}, u_{r}^{(j)}\right\} \cap F^{(j-1)}=\operatorname{sgp}\left\{u_{r}^{(0)}, \ldots, u_{r}^{(j-1)}\right\} \\
(j=1, \ldots, \lambda) .
\end{array}
$$

This follows from Proposition 1(i) if $j=\lambda$ (making use of Lemma 4). On the other hand if $j<\lambda$ then it follows from Lemma $1(\mathrm{i})$ since $u_{r}^{(j)} \in F^{(j)} \backslash F^{(j-1)}$ (see $\$ 2.2(\mathrm{~A}))$. Now

$$
\operatorname{sgp}\left\{a_{r}, c_{r}, d_{r}, \ldots\right\} \subseteq F^{(0)} \subset F^{(1)} \subset \cdots \subset F^{(\lambda)},
$$

and this together with (3.5) and (3.6) shows that $\operatorname{sgp}\left\{t^{p} h, u_{r}\right\} \cap \operatorname{sgp}\left\{a_{r}, c_{r}\right.$, $\left.d_{r}, \ldots\right\}=\operatorname{sgp}\left\{u_{r}^{(0)}\right\}$, as required.

This completes the proof of Proposition 2.

The following corollary of the proof will be needed in $\$ 4$.

COROllary. Suppose that $L$ does not have any generators $c_{i}(i \in \mathbf{Z})$, $d_{i}(i \in \mathbf{Z}), \ldots$, and that $N>0$. If $\left(v, a_{0}^{q}\right)$ generates $G$ then $|q|=1$ and $v$ or its inverse is expressible in the form $t h^{*}$, where $h^{*}$ is $t$-free. Moreover, if $N=1$ then $\left(a_{0}, h^{*-1} a_{1} h^{*}\right)$ generates $L$, whereas if $N>1$ there are integers $\alpha, \beta$ such that $h^{*}=a_{1}^{\alpha} a_{0}^{\beta}$.

Suppose $\left(v, a_{0}^{q}\right)$ generates $G$. Then $q \neq 0$ since $G$ is not cyclic. Taking $u \equiv a_{0}^{q}$ and following through the proof of Proposition 2 it can be seen that there are integers $m$ and $r$, with $r \geqslant 0$, such that $t^{-r}\left(v a_{0}^{m q}\right)^{\varepsilon} t^{r}=t^{p} h$ where 0 $<p \leqslant N-r, h$ is $t$-free, $h a_{r} h^{-1} \notin A_{1}$, and the standard $L$-elements $\left(a_{r}^{q}\right)^{(0)}$, $\ldots,\left(a_{r}^{q}\right)^{(\lambda)}$ generate $L$. By considering the factor group of $G$ by the normal subgroup generated by $L$ it is easily established that $p=1$. Also, since $L$ cannot be generated by less than $N+1$ elements, $\lambda=N, r=0$ and $h$ $\in \operatorname{sgp}\left\{a_{0}, a_{1}\right\}($ see $\$ 2.2(\mathbf{A}))$. 
Clearly if $N=1$ then $a_{0}$ and $h^{-1} a_{1} h$ must generate $L$. Also $\operatorname{sgp}\left\{a_{0}^{q}\right.$, $\left.h^{-1} a_{1}^{q} h\right\} \cap A_{-1}=\operatorname{sgp}\left\{a_{0}^{q}\right\}$ since $L$ has property-I, so that $|q|=1$.

Suppose on the other hand that $N>1$. Then $\left(h a_{0}^{q} h^{-1}, a_{1}^{q}, h^{-t} a_{2}^{q} h^{t}, \ldots\right.$, $\left.h^{-t}, \ldots, h^{-t^{N-1}} a_{N}^{q} h^{t^{N-1}} \cdots h^{t}\right)$ is weakly $\left(a_{0}, a_{N}\right)$-admissible and generates $L$. Thus (see [10, Corollary 3.1]) $h a_{0}^{q} h^{-1}=w a_{0}^{\delta} w^{\prime}$ where $w, w^{\prime} \in \operatorname{sgp}\left\{a_{1}, \ldots\right.$, $\left.a_{N-1}\right\}$ and $\delta= \pm 1$. Using the fact that $A_{-1}$ is freely generated by $a_{0}, \ldots$, $a_{N-1}$ it follows easily that $q=\delta$ and $h=a_{1}^{\kappa} a_{0}^{\theta}$ for suitable integers $\kappa, \theta$.

It now suffices to take $h^{*}=h a_{0}^{-q m}$ if $\varepsilon=1$ and $h^{*}=a_{1}^{q m} h$ if $\varepsilon=-1$.

3.3. A null-intersection lemma. Let $B=\left\langle x_{j}(j \in J) ; S, T, \ldots\right\rangle$ and for $j \in J$ define $L_{j}$ to be the subgroup of $B$ generated by those generators of $B$ other than $x_{j}$. Then $B$ (or more precisely this presentation of $B$ ) will be said to have property-NI provided the following holds: for each $j$ in $J$, if $u, v$ $\in L_{j}$ and $z \notin L_{j}$ then $z \operatorname{sgp}\left\{u, z^{-1} v z\right\} \cap L_{j}$ is empty.

The following lemma is needed for the proof of Proposition 3 in \$3.4.

LeMma 7. Let $B=\left\langle x, y, b, \ldots ; R^{n}\right\rangle$ where $R$ is cyclically reduced and $\left.n\right\rangle 1$. Then $B$ has property-NI.

The proof is by induction on the length of $R$.

If $R$ is empty then $B$ is freely generated by $x, y, b, \ldots$ and the result is easily established.

Now suppose that $L(R)>0$. Let $u, v$ be freely reduced words in $y, b, \ldots$, and suppose $z \notin \operatorname{sgp}\{y, b, \ldots\}$. It will be shown that $z \operatorname{sgp}\left\{u, z^{-1} v z\right\}$ $\cap \operatorname{sgp}\{y, b, \ldots\}$ is empty. This is trivial if either $u$ or $v$ is equal to 1 , so it suffices to consider the case when $u \neq 1, v \neq 1$ and show that it is impossible for an equation

$$
z u^{m_{1}} z^{-1} v^{n_{1}} z \cdots u^{m_{s}} z^{-1} v^{n_{s}} z=w
$$

where $s \geqslant 0$, the $\left|m_{i}\right|(i=1,2, \ldots, s)$ are greater than zero and less than the order of $u$, the $\left|n_{i}\right|(i=1,2, \ldots, s)$ are greater than zero and less than the order of $v, w$ is a word in $y, b, \ldots$ - to take place in $B$. It can be assumed that $u$ and $v$ are cyclically reduced. For suppose $u \equiv g u_{1} g^{-1}$ and $v \equiv h^{-1} v_{1} h$, where $u_{1}$ and $v_{1}$ are cyclically reduced. Let $z_{1}=h z g$. Then (3.7) is equivalent to

$$
z_{1} u_{1}^{m_{1}} z_{1}^{-1} v_{1}^{n_{1}} z_{1} \cdots u_{1}^{m_{s}} z_{1}=h w g .
$$

Case 1: $x$ does not occur in $R$.

Then $B$ is the free product of the free group on $x$ and the one-relator group generated by the remaining generators. The result is thus easily established using the theory of free products.

Case 2: No generator occurring in $u$ or $v$ also occurs in $R$.

Then $B$ is the free product of the free group $F$ on those generators occurring in one of $u, v$ with the one-relator group $B^{\prime}$ generated by the remaining 
generators. Now $z=f_{0} g_{1} f_{1} \cdots g_{l} f_{l}$ where $l>0$, the $f_{i}$ are elements of $F$, nontrivial except possibly for $f_{0}$ and $f_{l}$, the $g_{i}$ are nontrivial elements of $B^{\prime}$. Moreover, since $z \notin \operatorname{sgp}\{y, b, \ldots\}$ at least one of the $g_{i}$ does not belong to $\operatorname{sgp}\{y, b, \ldots\}$. Now the left-hand side of (3.7) is equal to

$$
\begin{array}{r}
f_{0} g_{1} f_{1} \cdots g_{l}\left(f_{l} u^{m_{1}} f_{l}^{-1}\right) g_{l}^{-1} \cdots f_{1}^{-1} g_{1}^{-1}\left(f_{0}^{-1} v^{n_{1}} f_{0}\right) g_{1} f_{1} \cdots g_{l} \\
\cdots\left(f_{l} u^{m_{s}} f_{l}^{-1}\right) g_{l}^{-1} \cdots f_{1}^{-1} g_{1}^{-1}\left(f_{0}^{-1} v^{n_{s}} f_{0}\right) g_{1} f_{1} \cdots g_{l} f_{l},
\end{array}
$$

and this latter is a normal form apart from trivial complications caused at the ends if $f_{0}$ or $f_{l}$ is equal to 1 . Since all terms of the normal form of $w$ belong to $\operatorname{sgp}\{y, b, \ldots\}$ it thus follows that (3.7) is impossible.

Case 3: $x$ occurs in $R$ with zero-sum exponent; one of $u, v$ involves a generator which occurs in $R$.

Suppose for definiteness that $y$ occurs in $u$ and $R$. Calculations will be done relative to the HNN presentation of $B$ with stable letter $x$ and fixed generator $y$.

Let $Z$ denote the $x$-reduced form of $z$. Then substituting into (3.7) gives

$$
Z u^{m_{1}} Z^{-1} v^{n_{1}} Z \cdots u^{m_{s}} Z^{-1} v^{n_{s}} Z=w .
$$

Now in order for this equation to take place, the $x$-reduced form of the lefthand side must be $x$-free. In particular $o_{x}(Z)=0$. Now by Lemmas 5 and 6 if $S$ is an initial segment of $Z$ such that $S^{-1} v^{n_{i}} S x$-reduces to an $x$-free word, then $S=x^{p} h_{1}$ for some integer $p$ and some $x$-free word $h_{1}$. Also, if $T$ is a terminal segment of $Z$ such that $T u^{m_{i}} T^{-1} x$-reduces to an $x$-free word then it follows from Lemmas 5, 6 and 3(i) that $T=h_{2} t^{-q}$ for some integer $q$ with $0 \leqslant q \leqslant M$, and some $x$-free word $h_{2}$. Consequently the only way (3.8) can hold is if $Z=t^{r} h t^{-r}$ where $0 \leqslant r \leqslant M$ and $h$ is $x$-free. But then (3.8) is equivalent to

$$
h u_{r}^{m_{1}} h^{-1} v_{r}^{n_{1}} h \cdots u_{r}^{m_{s}} h^{-1} v_{r}^{n_{s}} h=w_{r}
$$

Here $u_{r}, v_{r}, w_{r}$ are the words obtained from $u, v, w$ respectively by replacing $y_{0}$ by $y_{r}, b_{0}$ by $b_{r}, \ldots$ However since $h \notin \operatorname{sgp}\left\{y_{r}, b_{r}, \ldots\right\}$ this equation is impossible, for the base of $B$ has property-NI by the inductive hypothesis.

Case 4: $x$ occurs in $R$; one of $u, v$ involves a generator which occurs in $R$ with zero-sum exponent.

Suppose for definiteness that $y$ occurs in $u$ and $R$, and $\sigma_{y}(R)=0$. Calculations will be done relative to the HNN presentation of $B$ with stable letter $y$ and fixed generator $x$.

Now $z$ can be expressed in the form $y^{\theta} k y^{\rho}$ where $y^{\theta} k y^{\rho}$ is $y$-reduced and where $k$ is such that $k y^{ \pm 1}, y^{ \pm 1} k$ are all $y$-reduced. Then there are integers. 
$p, q, r$ and words $\bar{u}, \bar{v}, \bar{w}$ in $b_{i}(i \in \mathbf{Z}), \ldots$ such that $y^{\rho} u y^{-\rho}=y^{p} \bar{u}, y^{-\theta} v y^{\boldsymbol{\theta}}$ $=y^{q} \bar{v}, y^{-\theta} w y^{-\rho}=y^{r} \bar{w}$. Clearly (3.7) is equivalent to

$$
y^{-r} k\left(y^{p} \bar{u}\right)^{m_{1}} k^{-1}\left(y^{q} \bar{v}\right)^{n_{1}} k \cdots\left(y^{p} \bar{u}\right)^{m_{s}} k^{-1}\left(y^{q} \bar{v}\right)^{n_{s}} k=\bar{w} .
$$

Now in order for (3.9) to hold, the $y$-reduced form of the left-hand side must be $y$-free. This implies that $k$ is $y$-free. For suppose by way of contradiction that $k$ involves $y$. Then $k\left(y^{p} \bar{u}\right)^{m_{i}} k^{-1}$ is $y$-reduced for each $i$. This is clear if $p \neq 0$. Suppose on the other hand that $p=0$, and let $k$ have initial segment $g y^{\varepsilon}$ where $g$ is $y$-free. If $g^{-1} \bar{u}^{m_{i}} g \in K_{-\varepsilon}$ then $g \in K_{-\varepsilon}$ by (2.1) so that $y^{-\varepsilon} k$ is not $y$-reduced contrary to the definition of $k$. In a similar way $k^{-1}\left(y^{q} \bar{v}\right)^{n_{i}} k$ is $y$-reduced for each $i$. Thus the left-hand side of (3.9) is $y$-reduced and involves $y$, which is a contradiction.

Suppose that $k \in K_{-1} \cup K_{1}$. Conjugating (3.9) by a power of $y$ if necessary, it can be supposed that $k \in K_{-1} \backslash K_{1}$ (note that $k \notin \operatorname{sgp}\left\{b_{i}(i\right.$ $\in \mathbf{Z}), \ldots\})$. Now the set $\left\{b_{i}(i \in \mathbf{Z}), \ldots\right\}$ is closed under conjugation by $y$, so it follows from $\S 2.2(\mathbf{B})$ that $\operatorname{sgp}\{y, k, b, \ldots\}$ has presentation $\langle y, k, b, \ldots$; $\left.T^{n}(y, k, b, \ldots)\right\rangle$ where $T$ is cyclically reduced and is either empty or involves $y, k$.

Let $W$ denote an arbitrary word in the symbols $y, k, b, \ldots$ of the form

$$
w_{0} k w_{1} k^{-1} w_{2} k \cdots w_{2 \mu-1} k^{-1} w_{2 \mu} k
$$

where the $w_{i}$ are freely reduced words in $y, b, \ldots$ In order to show that (3.9) is impossible it suffices to establish that $W \neq 1$. The proof is by induction on $\mu$. If $\mu=0$ the result follows from Newman's Spelling Theorem. Suppose $\mu>0$. The only case requiring attention is when all of $w_{1}, w_{2}, \ldots, w_{2 \mu-1}, w_{2 \mu}$ are nonempty. Then if $W=1, T$ must be nonempty and $W$ must have a subword $\left(k^{\varepsilon} S\right)^{n-1} k^{\varepsilon}$ where $k^{\varepsilon} S$ is a cyclic permutation of $T^{ \pm 1}$ (see Statement 1, p. 1439 of [2]). Replacing this subword of $W$ by $S^{-1}$ and freely reducing the $k$-free subwords of the resulting word gives a word $W^{\prime}$ of the form (3.10) which is equal to $W$ and to which the inductive hypothesis applies. Thus $W^{\prime}$ $\neq 1$ so that $W \neq 1$.

Now suppose that $k \notin K_{-1} \cup K_{1}$. Then the left-hand side of (3.9) is $y$ reduced. This is clear except in the case when one of $p, q$ is nonzero and the other is zero. To deal with this case it suffices to observe that if $\bar{u}^{m}$ $\neq 1$ then $k \bar{u}^{m} k^{-1} \notin K_{-1} \cup K_{1}$, and if $\bar{v}^{m} \neq 1$ then $k^{-1} \bar{v}^{m} k \notin K_{-1} \cup K_{1}$ (this follows from (2.1)). Now since the $y$-reduced form of the the left-hand side of (3.9) must be $y$-free, $r=p=q=0$. But then equation (3.9) takes place in the base of $B$. However this is impossible since the base of $B$ has property-NI by the inductive hypothesis.

Case 5: $x$ occurs in $R ; \sigma_{x}(R) \neq 0$; one of $u, v$ involves a generator which occurs in $R$ with non zero-sum exponent. 
Suppose for definiteness that $y$ occurs in $u$ and $R$ and $\sigma_{y}(R) \neq 0$. Let $\alpha$ $=\sigma_{x}(R), \beta=\sigma_{y}(R)$. Let $B_{1}=\left\langle\bar{x}, \bar{y}, b, \ldots ; R_{1}^{n}\right\rangle$ where $R_{1}$ is obtained from $R$ by replacing each occurrence of $x$ by $\bar{x} \bar{y}^{-\beta}$ and each occurrence of $y$ by $\bar{y}^{\alpha}$, and cyclically reducing. Then $B$ is embedded into $B_{1}$ by the homomorphism $\Psi$ defined by

$$
x \mapsto \bar{x} \bar{y}^{-\beta}, \quad y \mapsto \bar{y}^{\alpha}, \quad b \mapsto b, \ldots
$$

Moreover:

$$
\Psi(B) \cap \operatorname{sgp}\{\bar{y}, b, \ldots\}=\operatorname{sgp}\left\{\bar{y}^{\alpha}, b, \ldots\right\} .
$$

Consequently $\Psi(z) \notin \operatorname{sgp}\{\bar{y}, b, \ldots\}$.

Now if $R_{1}$ involves both $\bar{x}$ and $\bar{y}$ then it follows as in Case 4 that $\Psi\left(z \operatorname{sgp}\left\{u, z^{-1} v z\right\}\right) \cap \operatorname{sgp}\{\bar{y}, b, \ldots\}$ is empty. On the other hand if $R_{1}$ does not involve $\bar{y}$ then $L\left(R_{1}\right)<L(R)$ so it follows from the inductive hypothesis that $\Psi\left(z \operatorname{sgp}\left\{u, z^{-1} v z\right\}\right) \cap \operatorname{sgp}\{\bar{y}, b, \ldots\}$ is empty. Thus in either situation it is easily seen that $z \operatorname{sgp}\left\{u, z^{-1} v z\right\} \cap \operatorname{sgp}\{y, b, \ldots\}$ is empty.

The above cases cover all possibilities and the induction step is proved.

3.4. Intersections of certain subgroups (3).

Proposition 3. Let $u$ be a nonempty freely reduced word in $t, c_{0}, d_{0}, \ldots$ and let $v$ be an element of $G$ which does not belong to $\operatorname{sgp}\left\{t, c_{0}, d_{0}, \ldots\right\}$. Then $\operatorname{sgp}\{u, v\} \cap \operatorname{sgp}\left\{t, c_{0}, d_{0}, \ldots\right\}=\operatorname{sgp}\{u\}$.

In order to prove this proposition it is of course necessary to determine which elements of $\operatorname{sgp}\{u, v\}$ are also elements of $\operatorname{sgp}\left\{t, c_{0}, d_{0}, \ldots\right\}$. Now an element of $\operatorname{sgp}\left\{t, c_{0}, d_{0}, \ldots\right\}$ can be expressed in the form $t^{-s} w$ where $w$ is a word in $c_{i}(i \in \mathbf{Z}), d_{i}(i \in \mathbf{Z}), \ldots$ (or alternatively in the form $w^{\prime} t^{-s}$ where $w^{\prime}$ is a word in $\left.c_{i}(i \in \mathbf{Z}), d_{i}(i \in \mathbf{Z}), \ldots\right)$. Consequently, a good deal of the proof of Proposition 3 will be concerned with determining whether for a given element $W$ of $\operatorname{sgp}\{u, v\}$ there is an integer $s$ such that $t^{s} W$ (or $W t^{s}$ ) belongs to $\operatorname{sgp}\left\{c_{i}(i \in \mathbf{Z}), d_{i}(i \in \mathbf{Z}), \ldots\right\}$.

Let $V$ be an element of minimal $t$-length from the set

$\left\{U: U\right.$ is a $t$-reduced word equal to $t^{-\alpha} u^{\gamma} v u^{\eta} t^{\alpha}$ for integers $\left.\alpha, \gamma, \eta\right\}$.

Then there are integers $\kappa, \beta, \omega$ such that $V=t^{-\kappa} u^{\beta} v u^{\omega} t^{\kappa}$. Moreover, it is not difficult to establish that there are integers $\theta, \rho$ such that $V=t^{\theta} z t^{\rho}$, where $t^{\theta} z t^{\rho}$ is $t$-reduced and where each of the words $t^{ \pm 1} z, z t^{ \pm 1}$ is $t$-reduced.

Now $t^{-(k-\rho)} u t^{\kappa-\rho}$ is equal in $G$ to a word $t^{m} k$ where $k$ is a word in $c_{i}(i \in \mathbf{Z}), d_{i}(i \in \mathbf{Z}), \ldots$. Let $p=\rho+\theta$. Then it suffices to show that $\operatorname{sgp}\left\{t^{p} z, t^{m} k\right\} \cap \operatorname{sgp}\left\{t, c_{0}, d_{0}, \ldots\right\}=\operatorname{sgp}\left\{t^{m} k\right\}$.

Case $1: z$ involves $t, p \neq 0$. 
Let $W$ denote a fixed but arbitrary word of the form

$$
\left(t^{p} z\right)^{q_{0}}\left(t^{m} k\right)^{l_{1}}\left(t^{p} z\right)^{q_{1}} \cdots\left(t_{m} k\right)^{l_{r}}\left(t^{p} z\right)^{q_{r}},
$$

where $r \geqslant 0$, and where the $q_{i}, l_{i}$ are nonzero integers. It suffices to establish that for no integer $s$ does $t^{s} W$ define an element of $L$. To prove this it is enough to show that in $t$-reducing $t^{s} W$ no $t$-symbol from any subword $z^{ \pm 1}$ is removed. This is easily deduced from the following remarks.

First note that the minimality of $V$ implies that in $t$-reducing $\left(t^{m} k\right)^{ \pm 1} t^{p} z$ no more than $[|m| / 2] t$-symbols from $\left(t^{m} k\right)^{ \pm 1}$ are used up, and the definition of $z$ implies that no $t$-symbols from $z$ are used up. Also, the definition of $z$ implies that $t^{p} z t^{m} k$ and $t^{p} z\left(t^{m} k\right)^{-1}$ are both $t$-reduced. It thus follows that if $m \neq 0$ then in $t$-reducing a word of the form $t^{p} z\left(t^{m} k\right)^{l} t^{p} z(l \neq 0)$ no $t$-symbols from either copy of $z$ are used up. This is also easily seen to be true if either $m=0$ or $l=0$ by the definition of $z$.

Secondly, observe that a word of the form $t^{p} z\left(t^{m} k\right)^{l} z^{-1} t^{-p}(l \neq 0)$ is $t$ reduced. This follows immediately from the definition of $z$ if $m \neq 0$. On the other hand suppose $m=0$, and let $z$ have terminal segment $t^{\varepsilon} h$, where $h$ is $t$ free. Now if $h k^{l} h^{-1} \in A_{\varepsilon}$ then $h \in A_{\varepsilon}$ by (2.1). Consequently $z t^{-e}$ is not $t$ reduced, which contradicts the definition of $z$.

Finally, consider $z^{-1} t^{-p}\left(t^{m} k\right)^{l} t^{p} z(l \neq 0)$. Now $t^{-p} t^{m} k t^{p}=t^{m} k^{\prime}$, where $k^{\prime}$ is a word in $c_{i}(i \in \mathbf{Z}), d_{i}(i \in \mathbf{Z}), \ldots$. Then an argument similar to that in the previous paragraph shows that $z^{-1}\left(t^{m} k^{\prime}\right)^{l} z$ is $t$-reduced.

Case 2: $z$ involves $t, p=0$.

It follows as in Case 1 that if $l$ is a nonzero integer then $z^{-1}\left(t^{m} k\right)^{l} z$ and $z\left(t^{m} k\right)^{l} z^{-1}$ are $t$-reduced.

Suppose that for every integer $j, z\left(t^{m} k\right)^{j} z$ is $t$-reduced. If $W$ is a word as in (3.11) and $s$ is an arbitrary integer then it is easily seen that $t^{s} W$ is $t$-reduced and therefore does not define an element of $\operatorname{sgp}\left\{c_{i}(i \in \mathbf{Z}), d_{i}(i \in \mathbf{Z}), \ldots\right\}$. Thus sgp $\left\{z, t^{m} k\right\} \cap \operatorname{sgp}\left\{t, c_{0} d_{0}, \ldots\right\}=\operatorname{sgp}\left\{t^{m}\right\}$, as required.

Suppose on the other hand that for some integer $j, z\left(t^{m} k\right)^{j} z$ is not $t$-reduced. Notice that, by the definition of $z$, this implies either $j=0$ or $m=0$. Let $Y$ be the cyclically $t$-reduced form of $z\left(t^{m} k\right)^{j}$. Then there is an initial segment $T$ of $z$, where $T$ has positive $t$-length, such that $z\left(t^{m} k\right)^{j}=T Y T^{-1}$ and $T Y T^{-1}$ is $t$-reduced. Since for every nonzero integer $l, z^{-1}\left(t^{m} k\right)^{l} z$ is $t$-reduced, it follows that $T^{-1}\left(t^{m} k\right)^{l} T$ is $t$-reduced. It is thus easy to see that if $X$ is a word of the form

$$
T Y^{q_{0}} T^{-1}\left(t^{m} k\right)^{l_{1}} T Y^{q_{1}} T^{-1} \cdots\left(t^{m} k\right)^{l_{r}} T Y^{q_{r}} T^{-1},
$$

where $r \geqslant 0$, the $\left|q_{i}\right|$ are nonzero and less than the order of $Y$, the $l_{i}$ are nonzero, then for every integer $s, t^{s} X$ is $t$-reduced and therefore does not 
define an element of $\operatorname{sgp}\left\{c_{i}(i \in \mathbf{Z}), d_{i}(i \in \mathbf{Z}), \ldots\right\}$. Thus $\operatorname{sgp}\left\{z\left(t^{m} k\right)^{j}, t^{m} k\right\}$ $\cap \operatorname{sgp}\left\{t, c_{0}, d_{0}, \ldots\right\}=\operatorname{sgp}\left\{t^{m} k\right\}$, as required.

Case 3: $z$ is $t$-free and defines an element of $A_{-1} \cup A_{1}$.

Conjugating the pair $\left(t^{p} z, t^{m} k\right)$ by a power of $t$ if necessary, it can be supposed that $z \in A_{-1} \backslash A_{1}$. Let $W$ denote an element of $\operatorname{sgp}\left\{t p_{z, t^{m}} k\right\}$, let $s$ be an integer, and let $w$ be a word in $c_{i}(i \in \mathbf{Z}), d_{i}(i \in \mathbf{Z}), \ldots$ It is required to determine when an equality

$$
t^{s} W=w
$$

can take place in $G$. To do this it is convenient to analyse $\operatorname{sgp}\{t, k, z\}$.

First suppose that $k \neq 1$, and let $\Re$ denote the set of standard $L$-elements associated with $(t, k)$. Then $\mathcal{K}$ is closed under conjugation by $t$ and the elements of $\mathcal{T}$ freely generate a subgroup of $\operatorname{sgp}\left\{c_{i}(i \in \mathbf{Z}), d_{i}(i \in \mathbf{Z}), \ldots\right\}$ (see the example of p. ). It therefore follows from (2.10) (with $\mu=-1$ ) that $\operatorname{sgp}\left\{c_{i}(i \in \mathbf{Z}), d_{i}(i \in \mathbf{Z}), \ldots\right\} \cap \operatorname{sgp}\{t, z\} \cup \mathcal{K}=\operatorname{sgp} \mathscr{K}$. Consequently if (3.12) holds then $w \in \operatorname{sgp} \Re$. Thus

$$
\begin{aligned}
\operatorname{sgp}\left\{t^{p} z, t^{m} k\right\} \cap \operatorname{sgp}\left\{t, c_{0}, d_{0}, \ldots\right\} & =\operatorname{sgp}\left\{t^{p} z, t^{m} k\right\} \cap \operatorname{sgp}\{t, k\} \\
& =\operatorname{sgp}\left\{t^{p} z, t^{m} k\right\} \cap \operatorname{sgp}\left\{t, t^{m} k\right\} .
\end{aligned}
$$

Now it follows from $\$ 2.2(\mathrm{~B})$ that $\operatorname{sgp}\{t, z, k\}$ has presentation $\left\langle t, z, k ; T^{n}\right\rangle$, where $T$ is either empty or is cyclically reduced and involves $t$ and $z$. Let $x=t^{p} z$ and $y=t^{m} k$. Then on the generators $t, x, y, \operatorname{sgp}\{t, z, k\}$ has presentation

$$
\left\langle t, x, y ; T_{1}^{n}\right\rangle
$$

where $T_{1}$ is obtained from $T$ by replacing each occurrence of $z$ by $t^{-p} x$ and each occurrence of $k$ by $t^{-m} y$, and cyclically reducing. Now using Newman's Spelling Theorem for the presentation (3.14) it can easily be shown that $\operatorname{sgp}\{x, y\} \cap \operatorname{sgp}\{t, y\}=\operatorname{sgp}\{y\}$. It therefore follows from (3.13) that $\operatorname{sgp}\{x$, y) $\cap \operatorname{sgp}\left\{t, c_{0}, d_{0}, \ldots\right\}=\operatorname{sgp}\{y\}$, as required.

There remains the situation when $k=1$. To deal with this situation proceed similarly as above, but take $\Re$ to be empty. The equation (3.13) is readily established. Moreover $\operatorname{sgp}\{t, z\}$, when presented on $t$ and $x\left(=t^{p} z\right)$, is a onerelator group where the relator when cyclically reduced is either empty or is an $n$th power which involves $x$. Consequently sgp $\left\{t^{m}, x\right\} \cap \operatorname{sgp}\{t\}=\operatorname{sgp}\left\{t^{m}\right\}$ by Newman's Spelling Theorem. It thus follows from (3.13) that $\operatorname{sgp}\left\{t^{m}, x\right\}$ $\cap \operatorname{sgp}\left\{t, c_{0}, d_{0}, \ldots\right\}=\operatorname{sgp}\left\{t^{m}\right\}$, as required.

Case 4: $z$ is t-free, $z \notin A_{-1} \cup A_{1}$. 
Subcase 4.1: $p=m=0$. Since $L$ has property-I it follows that $\operatorname{sgp}\{z, k\}$ $\cap A_{-1}=\operatorname{sgp}\{k\}$. Thus $\operatorname{sgp}\{z, k\} \cap \operatorname{sgp}\left\{c_{i}(i \in \mathbf{Z}), d_{i}(i \in \mathbf{Z}), \ldots\right\}=\operatorname{sgp}\{k\}$. Consequently $\operatorname{sgp}\{z, k\} \cap \operatorname{sgp}\left\{t, c_{0}, d_{0}, \ldots\right\}=\operatorname{sgp}\{k\}$, for it is clear that $\operatorname{sgp}\{z$, $k\} \cap \operatorname{sgp}\left\{t, c_{0}, d_{0}, \ldots\right\}=\operatorname{sgp}\{z, k\} \cap \operatorname{sgp}\left\{c_{i}(i \in \mathbf{Z}), d_{i}(i \in \mathbf{Z}), \ldots\right\}$.

Subcase 4.2: $p=0, m \neq 0$. It follows from (2.1) that if $q$ is an integer such that $z^{q} \neq 1$ then $z^{q} \notin A_{-1} \cup A_{1}$. Consequently, if $W$ is a word of the form

$$
z^{q_{0}}\left(t^{m} k\right)^{l_{1}} z^{q_{1}}\left(t^{m} k\right)^{l_{2}} z^{q_{2}} \cdots\left(t^{m} k\right)^{l_{r}} z^{q_{r}},
$$

where $r \geqslant 0, z^{q_{i}} \neq 1(i=0,1, \ldots, r), l_{i} \neq 0(i=1,2, \ldots, r)$, then for every integer $s, t^{s} W$ is $t$-reduced. Thus $W \notin \operatorname{sgp}\left\{t, c_{0}, d_{0}, \ldots\right\}$ so that $\operatorname{sgp}\left\{z, t^{m} k\right\}$ $\cap \operatorname{sgp}\left\{t, c_{0}, d_{0}, \ldots\right\}=\operatorname{sgp}\left\{t^{m} k\right\}$, as required.

Subcase 4.3: $p \neq 0, m=0$. It can be assumed that $p>0$. For $\operatorname{sgp}\left\{t^{p} z, k\right\}$ $=t^{p} \operatorname{sgp}\left\{t^{-p_{z}}{ }^{-1}, k^{t p}\right\} t^{-p}$, and $\operatorname{sgp}\left\{t^{p} z, k\right\} \cap \operatorname{sgp}\left\{t, c_{0}, d_{0}, \ldots\right\}=\operatorname{sgp}\{k\}$ if and only if $\operatorname{sgp}\left\{t^{-p} z^{-1}, k^{t p}\right\} \cap \operatorname{sgp}\left\{t, c_{0}, d_{0}, \ldots\right\}=\operatorname{sgp}\left\{k^{t p}\right\}$.

The result is easily established if $k=1$, so assume $k \neq 1$. Then $z k z^{-1}$ $\notin A_{1}$ by (2.1). Moreover if $k^{*}$ is the $t$-reduced form of $t^{-p} k t^{p}$ then $z^{-1} k^{*} z \notin A_{-1}$, again by (2.1). Consequently (see $\$ 2.2(\mathrm{~A})$ ), there are just two standard $L$-elements, namely $k$ and $z^{-1} k^{*} z$.

Suppose that $t^{s} w$ is an element of $\operatorname{sgp}\left\{t, c_{0}, d_{0}, \ldots\right\}$ which is equal to a word in $t^{p} z, k, z^{-1} k^{*} z$. Here $s$ is an integer and $w$ is a word in $c_{i}(i \in \mathbf{Z})$, $d_{i}(i \in \mathbf{Z}), \ldots$. Let $W$ be an element of minimal $t$-length from the set

$\left\{Z: Z\right.$ is a word in $t^{p} z, k, z^{-1} k^{*} z$, and $\left.Z=t^{s} w\right\}$.

Now by Britton's lemma the $t$-reduced form $\bar{W}$ of $W$ involves $|s| t$-symbols and all the exponents to which $t$ occurs in $\bar{W}$ have the same sign. It therefore follows from Proposition 1, Corollary that

$$
W \equiv Y_{1}\left(t^{p} z\right)^{\varepsilon} Y_{2}\left(t^{p} z\right)^{\varepsilon} \cdots Y_{r}\left(t^{p} z\right)^{\varepsilon} Y_{r+1}
$$

where $r \geqslant 0, \varepsilon r p=s$, the $Y_{i}$ are words in $k, z^{-1} k^{*} z$.

Now if $r=0$ then $Y_{1}=w$, so that $w$ is equal to a power of $k$. For

$$
\operatorname{sgp}\left\{k, z^{-1} k^{*} z\right\} \cap A_{-1}=\operatorname{sgp}\{k\},
$$

since $L$ has property-I.

In order to complete the proof that $\operatorname{sgp}\left\{t p_{z}, k\right\} \cap \operatorname{sgp}\left\{t, c_{0}, d_{0}, \ldots\right\}$ $=\operatorname{sgp}\{k\}$, it suffices to establish that $r \ngtr 0$. Suppose by way of contradiction that $r>0$, and assume for definiteness that $\varepsilon=1$. Then $Y_{1} \in A_{-1}$, so that $Y_{1}$ is equal to an element $k^{\mu}$ of $\operatorname{sgp}\{k\}$, by (3.15). Thus $k^{* \mu_{z}} Y_{2} \in A_{-1}$ (even if $r=1$ ). But this implies $z Y_{2} \in A_{-1}$, which contradicts the fact that $L$ has property-NI (see Lemma 7). 
Subcase 4.4: $p \neq 0, m \neq 0$. Replacing $t^{m} k$ by $t^{-m} k^{\prime}$ if necessary, where $k^{\prime}$ is the $t$-reduced form of $t^{m} k^{-1} t^{-m}$, it can be supposed that $m$ and $p$ have the same sign. Now the minimality of $V$ implies that in $t$-reducing $z^{-1} t^{-p} t^{m} k$ at most $[|m| / 2] t$-symbols from $t^{m} k$ are used up. Thus if $l=m-p$ then $l$ is nonzero and has the same sign as $m$. Consider the pair $t^{p} z, z^{-1} t^{l} k$. Then all four of the products $t^{p} z z^{-1} t^{l} k, t^{p} z k^{-1} t^{-l} z, z^{-1} t^{-p} z^{-1} t^{l} k, z^{-1} t^{-p} k^{-1} t^{-1} z$ are $t$-reduced, so it follows that a freely reduced word $W$ in $t^{p} z, z^{-1} t^{l} k$ is $t$ reduced. It must be ascertained whether $W$ can be equal to an element of $\operatorname{sgp}\left\{t, c_{0}, d_{0}, \ldots\right\}$. It will be shown by induction on the length of $W$ (as a word in $\left.t^{p} z, z^{-1} t^{l} k\right)$ that if $W$ defines an element of $\operatorname{sgp}\left\{t, c_{0}, d_{0}, \ldots\right\}$ then $W$ is a power of $t^{p} z z^{-1} t^{l} k\left(=t^{m} k\right)$.

The result is clear if $W$ is empty. Suppose $W$ is nonempty and that $W=t^{s} w$ where $w \in \operatorname{sgp}\left\{c_{i}(i \in \mathbf{Z}), d_{i}(i \in \mathbf{Z}), \ldots\right\}$. Then $t^{-s} W$ must $t$ reduce to a $t$-free word so that $W$ must have initial segment $t^{p} z$ or $k^{-1} t^{-l} z$. Suppose for example that $W \equiv t^{p} z W^{\prime}$. Then $W^{\prime}$ is nonempty, for $t^{p} z$ $\neq t^{s} w$ since $z \notin A_{-1} \cup A_{1}$. Thus $W^{\prime}$ can start with $t^{p} z, k^{-1} t^{-l} z$ or $z^{-1} t^{l} k$. In the former two cases however, $t^{-s+p} z W^{\prime}$ is $t$-reduced and therefore cannot be equal to $w$. In the latter case $W \equiv t^{p} z z^{-1} t^{l} k W^{\prime \prime}$, and $W^{\prime \prime}$ has shorter length than $W$ and defines an element of $\operatorname{sgp}\left\{t, c_{0}, d_{0}, \ldots\right\}$. Using the inductive hypothesis it is now concluded that $W$ is a power of $t^{p} z z^{-1} t^{l} k$, as required. The situation when $W$ has $k^{-1} t^{-l} z$ as initial segment is handled similarly.

The above cases cover all possibilities and the proof of Proposition 3 is now complete.

4. Proof of the Principal Lemma. In this section a proof of the Principal Lemma will be given (see \$4.2). Before doing this, however, it is recessary to solve the following problem: given a group $B$ with presentation $\left\langle x, y ; Q^{n}\right\rangle(n$ $>1$ ), for which elements $u$ do $x$ and $u$ together generate $B$ ? This problem is solved in $\$ 4.1$.

\subsection{Certain generating pairs of one-relator groups with torsion.}

LEMMA 8. Let $B=\left\langle x, y ; Q^{n}\right\rangle$ where $Q$ is cyclically reduced and involves $x$, and $n>1$. If $x$ is conjugate to $x y^{p}$ then $p=0$. If $x$ is conjugate to $x^{-1} y^{p}$ then either $p=0$ or $Q^{n}$ is a cyclic permutation of $\left(x y^{l}\right)^{ \pm 2}$, where $p=-2 l$.

Proof. The proof requires three case distinctions. Throughout the proof frequent use (without mention) will be made of Collins' lemma characterizing conjugacy in HNN groups (see [1, General Lemma 3]).

Case 1: $\sigma_{y}(Q)=0$.

Then every relator must have zero-sum exponent on $y$. Thus if $W^{-1} x W y^{-p} x^{-\varepsilon}$ is a relator for some word $W$ then $p=0$.

Case 2: $\sigma_{x}(Q)=0$. 
Then $Q$ involves both $x$ and $y$. Calculations will be done relative to the HNN presentation of $B$ with stable letter $x$ and fixed generator $y$.

Now if $x$ is conjugate to $x^{\varepsilon} y_{0}^{p}$ then $\varepsilon=1$ and there is a freely reduced word $u$ in the generators of the associated subgroup $K_{-1}$ of $B$ such that $u x u^{-1}$ $=x y_{0}^{p}$. Let $u^{*}$ be the word obtained from $u$ by replacing $y_{i}$ by $y_{i+1}$ for each generator $y_{i}$ appearing in $u$. Then $u^{*}$ is the $x$-reduced form of $x^{-1} u x$ and $u^{*}$ $=y_{0}^{p} u$. Now if $u$ is nonempty then $u^{*}$ is a word in the generators of $K_{1}$ and $u^{*}$ involves a generator of the base of $B$ which does not occur in $y_{0}^{p} u$. Thus $u^{*} \neq y_{0}^{p} u$ by Newman's Spelling Theorem. Consequently $u$ must be empty, so that $p=0$ as required.

Case 3: $\sigma_{x}(Q) \neq 0, \sigma_{y}(Q) \neq 0$.

Let $\sigma_{x}(Q)=\mu, \sigma_{y}(Q)=\eta$, and let $B_{1}=\left\langle c, d ; Q_{1}^{n}\right\rangle$, where $Q_{1}$ is obtained from $Q$ by replacing each occurrence of $x$ by $c d^{-\eta}$ and each occurrence of $y$ by $d^{\mu}$, and cyclically reducing. Then $B$ is embedded into $B_{1}$ by the homomorphism defined by $x \mapsto c d^{-\eta}, y \mapsto d^{\mu}$.

Consider first the situation when $Q_{1}$ involves both $c$ and $d$. Suppose that $c d^{-\eta}$ and $\left(c d^{-\eta}\right)^{\varepsilon} d^{p \mu}$ are conjugate in $B_{1}$. If $\varepsilon=1$ then $p=0$ since $\sigma_{d}\left(Q_{1}\right)$ $=0$. Suppose $\varepsilon=-1$. Then $\eta+p \mu=-\eta$ and $c d^{-\eta}$ is conjugate to $c^{-1} d^{-\eta}$. It can be assumed without loss of generality that $\mu$ is negative. The calculations in the next three paragraphs will be done with respect to the HNN presentation of $B_{1}$ with stable letter $d$ and fixed generator $c$.

Now if $c d^{-\eta}$ and $c^{-1} d^{-\eta}$ are conjugate then there is a freely reduced word $u$ in the generators of the associated subgroup $K_{-1}$ of $B_{1}$ such that

$$
d^{\kappa} c_{0}^{\delta} d^{\theta}=u^{-1} d^{\rho} c_{0}^{-\delta} d^{\tau} u,
$$

where $\kappa, \rho>0, \theta, \tau \geqslant 0, \delta= \pm 1, \kappa+\theta=\rho+\tau=-\eta$. Moreover, it is no loss of generality to assume that $\kappa \geqslant \rho$ (so that $\tau \geqslant \theta$ ).

Suppose first that $u$ is nonempty and let $q$ and $s$ be respectively the least and greatest integers $i$ for which $c_{i}$ occurs in $u$. Let $u_{j}(-q \leqslant j \leqslant M-s)$ be the word obtained from $u$ by replacing each generator $c_{i}$ appearing in $u$ by $c_{i+j}$ (so that $u_{j}$ is the $d$-reduced form of $\left.d^{-j} u d^{j}\right)$. Now if exactly $2 r d$-symbols are used up in $d$-reducing $d^{\tau} u d^{-\theta}$ then it follows from Lemma 3 that $r \leqslant q$ and $d^{\tau} u d^{-\theta}$ $=d^{\tau-r} u_{-r} d^{-(\theta-r)}$. Thus

$$
d^{-\kappa} u^{-1} d^{\rho} c_{0}^{-\delta} d^{\tau-r} u_{-r} d^{-(\theta-r)}=c_{0}^{\delta} .
$$

In order for this equation to hold all of $d^{\rho}$ must be used up in $d$-reducing $d^{-\kappa} u^{-1} d^{\rho}$. Consequently $0 \leqslant \rho \leqslant M-s$ and $d^{-\kappa} u^{-1} d^{\rho}=d^{-(\kappa-\rho)} u_{\rho}^{-1}$, again by Lemma 3 . Thus

$$
d^{-(\kappa-\rho)} u_{\rho}^{-1} c_{0}^{-\delta} d^{\tau-r} u_{-r} d^{-(\theta-r)}=c_{0}^{\delta} .
$$


Now if $r$ were less than $\theta$ then $\tau-r>\kappa-\rho$ so that the $d$-reduced form of the left-hand side of (4.1) would involve $d$, contrary to Britton's lemma. Thus $r=\theta$ and $\kappa-\rho=\tau-r$. Moreover $d^{-(\kappa-\rho)} u_{\rho}^{-1} c_{0}^{-\delta} d^{\tau-r}$ must belong to the base. Now if $\kappa-\rho>0$ then $d^{-(\kappa-\rho)} u_{\rho}^{-1} c_{0}^{-\delta} d^{\rho-r}$ can belong to the base only if $u_{\rho}^{-1}$ belongs to the associated subgroup $K_{-1}$, and so $\rho+s<M$ by (2.2). Consequently $\kappa-\rho \leqslant M-(\rho+s)$ and $d^{-(\kappa-\rho)} u_{\rho}^{-1} c_{0}^{-\delta} d^{\tau-r}=u_{\kappa}^{-1} c_{\kappa-\rho}^{-\delta}$ by Lemma 3. It therefore follows from (4.1) that if $\kappa-\rho>0$ then

$$
u_{\kappa}=c_{\kappa-\rho}^{-\delta} u_{-\theta} c_{0}^{-\delta} \text {. }
$$

This is also clearly valid if $\kappa-\rho=0$. But $u_{\kappa}$ is a freely reduced word in the generators of $K_{1}$ and $u_{k}$ involves a generator which does not occur in $c_{\kappa-\rho}^{-\delta} u_{-\theta} c_{0}^{-\delta}$. Thus (4.2) is impossible by Newman's Spelling Theorem.

Now suppose that $u$ is empty. Then $d^{\kappa-\rho} c_{0}^{\delta}=c_{0}^{-\delta} d^{\kappa-\rho}$. However this is impossible. For if $\kappa>\rho$ it would require $c_{0}^{\delta}$ to belong to $K_{1}$ contrary to (2.2), whereas if $\kappa=\rho$, it would assert that $c_{0}^{2}=1$, when in fact $c_{0}$ has infinite order.

It has now been established that if $Q_{1}$ involves both $c$ and $d$ then $c d^{-\eta}$ and $\left(c d^{-\eta}\right)^{\varepsilon} d^{p \mu}$ are conjugate only if $\varepsilon=1$ and $p=0$.

There remains the situation when $Q_{1}$ does not involve $d$. This can happen only if $\eta=l \mu$ for some integer $l$, and $Q$ is a cyclic permutation of $\left(x y^{l}\right)^{\mu}$. Let $b=x y^{l}$. Then $B=\left\langle b, y ; b^{\mu n}\right\rangle$. Suppose $b y^{-l}$ and $\left(b y^{-l}\right)^{\varepsilon} y^{p}$ conjugate in $B$. Clearly if $\varepsilon=1$ then $p=0$. On the other hand, if $\varepsilon=-1$ then it follows from the solution to the conjugacy problem for free products that $b^{2}=1$ and $p$ $=-2 l$.

LEMMA 9. Let $B=\left\langle x, y ; Q^{n}\right\rangle$ where $Q$ is cyclically reduced and involves $x$, and $n>1$. If $g^{-1} x g=x^{\varepsilon} y^{p}$ then there are integers $\alpha, \beta$ such that $g=x^{\alpha} y^{\beta}$.

Proof. If $p=0$ then $g \in \operatorname{sgp}\{x\}$ since sgp $\{x\}$ is malnormal in $B$ (see [8, Lemma 2.1]).

Suppose $p \neq 0$. Then it follows from Lemma 8 that $\varepsilon=-1, B=\langle x, y$; $\left(x y^{l}\right)^{2}>$ and $p=-2 l$. Thus

$$
y^{-l} g^{-1} x g y^{l}=y^{-l} x^{-1} y^{-l}=x .
$$

Consequently $g y^{l} \in \operatorname{sgp}\{x\}$ by malnormality.

LEMMA 10. Let $B=\left\langle x, y ; Q^{n}\right\rangle$ where $\left.n\right\rangle 1$, and where $Q$ is a nonempty cyclically reduced word which is not a true power. If $(x, u)$ generates $B$ then $u$ is expressible in the form $x^{\alpha} y^{\varepsilon} x^{\beta}$ for certain integers $\alpha, \beta$, unless some cyclic permutation of $Q^{ \pm 1}$ has the form $y x^{l}$.

Proof. Perhaps somewhat surprisingly, the proof is by induction on the length of $Q$. 
If $Q$ has length 1 then $B$ is the free product of its cyclic subgroups $\operatorname{sgp}\{x\}$ and $\operatorname{sgp}\{y\}$, and the result follows easily using the theory of free products.

Now assume that $Q$ has length greater than 1 (so that $Q$ involves $x$ and $y$ ), and suppose $(x, u)$ generates $B$. There are several cases to consider.

Case 1: $\sigma_{x}(Q)=0$.

Calculations will be done relative to the HNN presentation of $B$ with stable letter $x$ and fixed generator $y$. Notice that the base of $B$ has property-I by Theorem 4, and so the results of $\$ \S 3.1,3.2$ apply.

Now there are integers $\theta, \rho$ and an $x$-reduced word $w$ such that $u=x^{\theta} w x^{\rho}$, where $x^{\theta} w x^{\rho}$ is $x$-reduced and where each of the words $x^{ \pm 1} w, w x^{ \pm 1}$ is $x$ reduced. Since $(x, w)$ generates $B$ it is clear that $w \neq 1$. Also, $w$ must be $x$-free, for if $w$ involved $x$ then $\operatorname{sgp}\{x, w\}$ would intersect the base $H$ of $B$ trivially. Moreover $w \in K_{-1} \cup K_{1}$, for if $w \notin K_{-1} \cup K_{1}$ then using the fact that $K_{-1}$ and $K_{1}$ are malnormal in $H$ it is not difficult to show that $\operatorname{sgp}\{x, w\} \cap H$ $=\operatorname{sgp}\{w\}$, which is a contradiction since $H$ is not cyclic. Conjugating the pair $(x, w)$ by a power of $x$ if necessary it can be supposed that $w \in K_{-1} \backslash K_{1}$. Then the standard $H$-elements $w^{(0)}, \ldots, w^{(\lambda)}$ generate $H$ by Proposition 1(iii), so that $\lambda=M$. Thus $w=y_{0}^{q}$ for some integer $q$ by Lemma 3 , and $|q|=1$ by Proposition 2, Corollary.

Case 2: $\sigma_{y}(Q)=0$.

Calculations will be done relative to the HNN presentation of $B$ with stable letter $y$ and fixed generator $x$. Notice that the base of $B$ has property-I by Theorem 4 , and so the results of $\$ 3.2$ apply.

If the number of generators of the base $H$ of $B$ is more than 2 then it follows from Proposition 2, Corollary that $u=x_{0}^{\alpha} y^{\varepsilon} x_{0}^{\beta}$ for suitable integers $\alpha, \beta$.

Suppose, on the other hand, that $H$ is generated by $x_{0}$ and $x_{1}$. Then again by Proposition 2, Corollary either $u$ or its inverse is expressible in the form $y h$, where $h \in H$ and $\left(x_{0}, h^{-1} x_{1} h\right)$ generates $H$. Consequently by the inductive hypothesis either $h^{-1} x_{1} h=x_{0}^{\kappa} x_{1}^{\delta} x_{0}^{\mu}$ for certain integers $\kappa, \delta, \mu$ with $\delta= \pm 1$, or $H=\left\langle x_{0}, x_{1} ;\left(x_{1} x_{0}^{l}\right)^{n}\right\rangle$ for some nonzero integer $l$. In the former situation it follows from Lemma 9 that there are integers $p, r$ such that $h=x_{1}^{p} x_{0}^{r}$. This is also true in the latter situation (see [9, p. ]). Thus in either situation $u$ is expressible in the form $x_{0}^{\alpha} y^{\varepsilon} x_{0}^{\beta}$.

Case 3: $\sigma_{x}(Q) \neq 0, \sigma_{y}(Q) \neq 0$.

Let $\sigma_{x}(Q)=\eta, \sigma_{y}(Q)=\mu$ and let $B_{1}=\left\langle c, d ; Q_{1}^{n}\right\rangle$, where $Q_{1}$ is obtained from $Q$ by replacing each occurrence of $x$ by $d^{\mu}$ and each occurrence of $y$ by $c d^{-\eta}$ and cyclically reducing. Then $B$ is embedded into $B_{1}$ by the homomorphism $\Psi$ defined by $x \mapsto d^{\mu}, y \mapsto c d^{-\eta}$. Let $u^{\prime}=\Psi(u)$. Then $\left(d, u^{\prime}\right)$ generates $B_{1}$. For $c d^{-\eta}$ can be obtained from $d^{\mu}$ and $u^{\prime}$, so that $c$ can be obtained from $d$ and $u^{\prime}$. Now if $Q_{1}$ involves $c$ and $d$ then it follows as in Case 1 that $u^{\prime}$ or its 
inverse is expressible in the form $d^{\kappa} c d^{-\eta} d^{\rho}$. Since $u^{\prime} \in \Psi(B), \mu$ must divide $\kappa$ and $\rho$ so that $u$ is expressible in the form $x^{\alpha} y^{\varepsilon} x^{\beta}$, as required.

Suppose that $Q_{1}$ does not involve $d$. Then $\eta=l \mu$ for some integer $l$, and either $Q$ or its inverse is a cyclic permutation of $\left(y x^{l}\right)^{\eta}$ (thus $|\eta|=1$ ).

This completes the proof of the lemma.

4.2. Proof of the Principal Lemma. Let $G=\left\langle a, t ; R^{n}\right\rangle$ where $R$ is cyclically reduced and not a true power, and $n>1$. Now it follows from Lemma 4.1 of [11] that there is an automorphism $\Psi$ of the free group $F$ on $a, t$ such that $\Psi(R)$ has zero-sum exponent on $t$, and it is easily seen that $G=\left\langle a, t ; \Psi(R)^{n}\right\rangle$. Moroever, it follows from Corollary N4 of [3] that the cyclically reduced form of $\Psi(R)$ is a nontrivial power of $a$ (in which case it is $a^{ \pm 1}$ ) if and only if $R$ is a primitive. In order to determine the Nielsen equivalence classes of $G$ it can be assumed without loss of generality that $\Psi$ is the identity, so that $\Psi(R)=R$.

First note that if $R$ is empty then trivially $G$ has one Nielsen equivalence class.

Next suppose that $R \equiv a^{ \pm 1}$. Then it follows from the Grushko-Neumann Theorem that every generating pair of $G$ is Nielsen equivalent to a pair of the form $\left(a^{\alpha}, t\right)$ where $\alpha$ is coprime to $n$. Consequently $G$ has one Nielsen equivalence class if $n=2$. On the other hand, suppose $n>2$. Then two pairs $\left(a^{\alpha_{1}}, t\right),\left(a^{\alpha_{2}}, t\right)$, where $\alpha_{1}$ and $\alpha_{2}$ are coprime to $n$, are Nielsen equivalent if and only if $a^{\alpha_{1}}=a^{ \pm \alpha_{2}}$. For it follows from Theorem 3.9 of [3] that $\left(a^{\alpha_{1}}, t\right)$ and $\left(a^{\alpha_{2}}\right.$, $t)$ are Nielsen equivalent only if $a^{-\alpha_{1}} t^{-1} a^{\alpha_{1}} t$ is conjugate to $\left(a^{-\alpha_{2}} t^{-1} a^{\alpha_{2}} t\right)^{ \pm 1}$. Such a conjugacy can only take place if $a^{\alpha_{1}}=a^{ \pm \alpha_{2}}$ by Theorem 4.2 of [3]. This establishes that $G$ has $\frac{1}{2} \varphi(n)$ Nielsen equivalence classes. The fact that it has one $T$-system follows from the observation that the mapping $a \mapsto a^{\alpha}, t \mapsto t$, where $\alpha$ is coprime to $n$, defines an automorphism of $G$.

Now suppose that $R$ involves $a$ and $t$. By assumption $\sigma_{t}(R)=0$. Calculations will mainly be done with reference to the HNN presentation of $G$ with stable letter $t$ and fixed generator $a$.

It follows from Theorem 6 of [9] that every generating pair of $G$ is Nielsen equivalent to a pair of the form $(t h, k)$ where $h$ belongs to the base $H$ of $G$ and $k$ is a nontrivial element of the associated subgroup $K_{-1}$. Conjugating the pair $(t h, k)$ by an element of $K_{-1}$ if necessary it can be supposed that $k$ is a nonempty cyclically reduced word in the generators of $K_{-1}$. Moreover, conjugating the pair $(t h, k)$ by a power of $t$ if necessary, it can be assume that $h k h^{-1} \notin K_{1}$ (see $\$ 2.2(\mathrm{~A})$ ).

Let $k^{(0)}, \ldots, k^{(\lambda)}$ be the standard $H$-elements associated with $(t h, k)$. Now $H$ has property I by Theorem 4, and so it follows from Proposition 1(iii) that if $t h$ and $k$ generate $G$ then $k^{(0)}, \ldots, k^{(\lambda)}$ generate $H$. But $H$ cannot be generated by less than $M+1$ elements so that $\lambda=M$. Thus $t^{-M} k t^{M} \in H$ (see $\$ 2.2(\mathrm{~A}))$ and so $k \equiv a_{0}^{q}$ for some integer $q$, by Lemma 3. Moreover, it 
follows from Proposition 2, Corollary that $|q|=1$. Finally, the fact that $\left(t h, a_{0}\right)$ generates $G$ implies that there are integers $\alpha, \beta$ such that $t h=a_{0}^{\alpha} t a_{0}^{\beta}$. This follows from Lemma 10 by reverting back to the one-relator presentation of $G$.

It has now been established that every generating pair of $G$ is Nielsen equivalent to a pair of the form $\left(a^{\alpha} t a^{\beta}, a^{\varepsilon}\right)$. Since such a pair $\left(a^{\alpha} t a^{\beta}, a^{\varepsilon}\right)$ is obviously Nielsen equivalent to $(t, a)$ it follows that $G$ has one Nielsen equivalence class, as required.

This completes the proof of the Principal Lemma.

\section{REFERENCES}

1. D. J. Collins, Recursively enumerable degrees and the conjugacy problem, Acta Math. 122 (1969), 115-160. MR 39 \#4001.

2. G. A. Gurevič, On the conjugacy problem for groups with one defining relation, Dokl. Akad. Nauk SSSR 207 (1972), 18-20 = Soviet Math. Dokl. 13 (1972), 1436-1439. MR 47 \#5123.

3. W. Magnus, A. Karrass and D. Solitar, Combinatorial group theory: Presentations of groups in terms of generators and relations, Pure and Appl. Math., vol. 13, Interscience, New York, 1966. MR 34 \# 7617.

4. J. McCool, Some finitely presented subgroups of the automorphism group of a free group, J. Algebra 35 (1975), 205-213.

5. J. McCool and P. Schupp, On one relator groups and HNN extensions, J. Austral. Math. Soc. 16 (1973), 249-256. MR 49 \#2952.

6. D. I. Moldavanskiī, Certain subgroups of groups with one defining relator, Sibirsk. Mat. Ž. 8 (1967), 1370-1384 = Siberian Math. J. 8 (1967), 1039-1048. MR 36 \#3862.

7. B. B. Newman, Some results on one-relator groups, Bull. Amer. Math. Soc. 74 (1968), 568-571. MR 36 \# 5204.

8. - The soluble subgroups of a one-relator group with torsion, J. Austral. Math. Soc. 16 (1973), 278-285. MR 49 \#2954.

9. S. J. Pride, On the generation of one-relator groups, Trans. Amer. Math. Soc. 210 (1975), 331-364.

10. Certain subgroups of certain one-relator groups, Math. Z. 146 (1976), 1-6.

11. G. S. Sacerdote and P. E. Schupp, SQ-universality in $H N N$ groups and one relator groups, J. London Math. Soc. (2) 7 (1974), 733-740.

Faculty of Mathematics, The Open University, Milton Keynes, England 